\title{
DUALITY THEORY FOR COVARIANT SYSTEMS
}

BY

\author{
MAGNUS B. LANDSTAD
}

\begin{abstract}
If $(A, \rho, G)$ is a covariant system over a locally compact group $G$, i.e. $\rho$ is a homomorphism from $G$ into the group of *-automorphisms of an operator algebra $A$, there is a new operator algebra $\mathfrak{U}$ called the covariance algebra associated with $(A, \rho, G)$. If $A$ is a von Neumann algebra and $\rho$ is $\sigma$-weakly continuous, $\mathfrak{A}$ is defined such that it is a von Neumann algebra. If $A$ is a $C^{*}$-algebra and $\rho$ is norm-continuous $\mathfrak{A}$ will be a $C^{*}$-algebra. The following problems are studied in these two different settings: 1. If $\mathfrak{A}$ is a covariance algebra, how do we recover $A$ and $\rho$ ? 2 . When is an operator algebra $\mathfrak{A}$ the covariance algebra for some covariant system over a given locally compact group $G$ ?
\end{abstract}

Introduction. If $G$ is a locally compact group and $\rho: G \rightarrow \operatorname{Aut}(A)$ is a continuous homomorphism of $G$ into the group of ${ }^{*}$-automorphisms of an operator algebra $A$, the triple $(A, \rho, G)$ is called a covariant system. (A more precise definition is given in Chapters 2 and 3.) This notation was introduced by Doplicher, Kastler and Robinson in 1966, [10], but already Murray and von Neumann considered special cases with $A$ abelian and $G$ discrete in constructing the first non-type I factors. Covariant systems have turned out to be very interesting objects both in theoretical physics and in mathematics.

With a representation of $(A, \rho, G)$ we shall mean a pair $(S, U)$ consisting of a unitary representation $U$ of $G$ and a *-representation $S$ of $A$ with $S$ and $U$ operating over the same Hilbert space such that

$$
S_{\rho_{x}(a)}=U_{x} S_{a} U_{x^{-1}} \text { for } a \in A, x \in G .
$$

Doplicher, Kastler and Robinson showed that the representation theory of $(A, \rho, G)$ was essentially the same as that of a certain operator algebra $\mathfrak{A}$ called the covariance algebra of $(A, \rho, G)$. The representation theory of $\mathfrak{U}$ has been extensively studied by M. Takesaki in [22], G. Zeller-Meier in [30] and E. G. Effros and F. Hahn in [11] among others, and the covariance algebras provide us with a rich variety of examples of operator algebras. For instance many examples of factors are obtained this way (cf. [20, Chapter 4.2] for some), and A. Connes and M. Takesaki have recently shown that all type III

Received by the editors March 13, 1975.

AMS (MOS) subject classifications (1970). Primary Primary 46L05, 46L10.

Key words and phrases. Operator algebra, covariant system, covariance algebra. 
von Neumann algebras can be obtained as the covariance algebra of a covariant system $(A, \rho, \mathbf{R})$ over the real numbers with $A$ a type II von Neumann algebra (cf. [6] and [28]).

Before we proceed to describe the scope of this work, we shall make some more precise definitions. There are two kinds of covariant systems we are going to consider, first systems $(A, \rho, G)$ where $A$ is a von Neumann algebra and $\rho$ is $\sigma$-weakly continuous (i.e. the map $x \rightarrow \phi\left(\rho_{x}(a)\right)$ is continuous for all $a \in A$ and all normal functionals $\phi$ of $A$ ). In this case the covariance algebra $\mathfrak{U}$ is defined such that it is a von Neumann algebra. Second, we shall study systems $(A, \rho, G)$ where $A$ can be any $C^{*}$-algebra, but $\rho$ is now supposed to be norm-continuous (i.e. $x \rightarrow \rho_{x}(a)$ is norm-continuous for all $a \in A$ ). The covariance algebra will then be a $C^{*}$-algebra, but $\mathfrak{A}$ is usually not $\sigma$-closed.

Let us now concentrate on the von Neumann algebra situation, so let $(A, \rho, G)$ be a covariant system with $\rho \sigma$-continuous. If we think of $A$ as a concrete von Neumann algebra on a Hilbert space $H$ we can define the so-called regular representation $(\tilde{\rho}, \lambda)$ of $(A, \rho, G)$ over $X=L^{2}(G, H)$ by

$$
\begin{aligned}
\tilde{\rho}(a) f(x) & =\rho_{x^{-1}}(a) f(x), \\
\lambda(y) f(x) & =f\left(y^{-1} x\right) \quad \text { for } a \in A, f \in X, x, y \in G .
\end{aligned}
$$

The von Neumann algebra generated by the set $\{\tilde{\rho}(a) \lambda(x) \mid a \in A, x \in G\}$ will be called the regular covariance algebra of $(A, \rho, G)$. The main subject of Chapter 2 is to describe all von Neumann algebras which can be obtained as the regular covariance algebra of a covariant system $(A, \rho, G)$ over a given group $G$.

To get an impression of the ideas involved, let us look at what happens when $G$ is abelian. It then turns out that if $\mathfrak{A}$ is the regular covariance algebra of $(A, \rho, G)$, there is a natural $\sigma$-continuous homomorphism $\tau$ of the dual group $\hat{G}$ onto Aut $(\mathfrak{A})$ given by

$$
\tau_{\gamma}(a)=U_{\gamma} a U_{-\gamma} \text { for } a \in \mathfrak{A}, \gamma \in \hat{G},
$$

where $U_{\gamma}$ is the unitary operator over $X$ defined by

$$
U_{\gamma} f(x)=\gamma(x) f(x) \text { for } \gamma \in \hat{G}, f \in X, x \in G .
$$

It is easy to check that

$$
\tau_{\gamma}(\lambda(x))=\gamma(x) \lambda(x) \text { for } \gamma \in \hat{G}, x \in G .
$$

The duality theorem for covariance algebras over an abelian group (Theorem 2) then tells us that a von Neumann algebra $\mathfrak{A}$ is isomorphic to the regular covariance algebra of a covariant system $(A, \rho, G)$ over a given abelian group $G$ if and only if there are homomorphisms $\lambda: G \rightarrow \mathfrak{A}$ and $\tau: \hat{G} \rightarrow \operatorname{Aut}(\mathfrak{H})$ such that (0.4) holds. Furthermore, for a given $G,(A, \rho, G)$ is uniquely determined 
up to isomorphisms by $A=\left\{a \in \mathfrak{A} \mid \tau_{\gamma}(a)=a\right.$ for all $\left.\gamma \in \hat{G}\right\}$ and $\rho_{x}(a)=$ $\lambda(x) a \lambda\left(x^{-1}\right)$ for $x \in G, a \in A$.

To get some idea of the method of proof let us first give a sketch of the main part of the proof when the group $G$ is abelian.

So suppose we have a von Neumann algebra $\mathfrak{A}$ over a Hilbert space $A$ together with continuous homomorphisms $\tau: \hat{G} \rightarrow \operatorname{Aut}(\mathfrak{A})$ and $\lambda: G \rightarrow \mathfrak{A}$ such that $\tau_{\gamma}(\lambda(x))=\gamma(x) \lambda(x)$ for $x \in G, \gamma \in \hat{G}$. Let

$$
\mathfrak{A}_{0}=\left\{a \in \mathfrak{A} \mid \exists K_{a} \geqslant 0, \int_{\hat{G}}\left\|\tau_{\gamma}(a) \xi\right\|^{2} d \gamma \leqslant K_{a}\|\xi\|^{2} \text { for all } \xi \in H\right\}
$$

and take $\mathfrak{A}_{1}=\mathfrak{U}_{0}^{*} \mathfrak{H}_{0}$. Then $\mathfrak{U}_{0} \subset \mathfrak{A}_{0}$ and since $\lambda(f) \in \mathfrak{A}_{0}$ for $f \in C_{00}(G), \mathfrak{A}_{0}$ and $\mathfrak{A}_{1}$ will both be $\sigma$-dense in $\mathfrak{A}$. Let $A=\left\{a \in \mathfrak{A} \mid \tau_{\gamma}(a)=a\right.$ for all $\left.\gamma \in \hat{G}\right\}$ and define a linear map $P: \mathfrak{A}_{1} \rightarrow A$ by the formula

$$
\langle P(a) \xi, \eta\rangle=\int_{\hat{G}}\left\langle\tau_{\gamma}(a) \xi, \eta\right\rangle d \gamma .
$$

We want to prove that $(A \cup \lambda(G))^{\prime \prime}=\mathfrak{A}$, and in order to do this it suffices to prove that any element $a \in \mathfrak{A}_{1}$ can be approximated with elements from $(\lambda(G) \cup A)^{\prime \prime}$. This can be done as follows: Let $\left\{\varphi_{i}\right\}$ be a net from $L^{1}(G)$ such that the net $\left\{\hat{\varphi}_{i}\right\}$ of Fourier transforms is an approximate identity in $L^{1}(\hat{G})$ (this is possible, cf. $[19,1.6 .4])$. Then take $a_{i}=\int_{G} \varphi_{i}(x) P(a \lambda(x)) \lambda\left(x^{-1}\right) d x$. $a_{i} \in(A \cup \lambda(G))^{\prime \prime}$ and $a_{i} \rightarrow a$ in the $\sigma$-topology. So $\mathfrak{A}=(A \cup \lambda(G))^{\prime \prime}$ and if we define $\rho_{x}(a)=\lambda(x) a \lambda\left(x^{-1}\right)$ for $a \in A, x \in G$ it is not too difficult to prove that $\mathfrak{U}$ is isomorphic to the covariance algebra of $(A, \rho, G)$. In order to state a duality theorem for regular covariance algebras over a nonabelian group $G$, we immediately see that we need a duality theorem for nonabelian groups. Takesaki showed that all the apparently different duality theorems for nonabelian locally compact groups can be given a unified approach by introducing so called "involutive abelian Hopf-von Neumann algebras", see [26]. Let us give a brief sketch of the ingredients of this theory. If $\mathcal{L}(G)$ is the von Neumann algebra generated by the left regular representation of $G$ over $L^{2}(G)$ we can define a ${ }^{*}$-isomorphism $\delta_{G}: \mathcal{L}(G) \rightarrow \mathcal{L}(G) \otimes \mathcal{L}(G)$ by

$$
\delta_{G}(a)=V^{*}(a \otimes I) V \quad \text { for } a \in \mathcal{L}(G)
$$

where $V$ is the unitary operator over $L^{2}(G \times G)$ defined by $V f(s, t)=$ $f(s, s t)$. If $i$ is the identity map of $\mathcal{L}(G)$ we will have

$$
\left(i \otimes \delta_{G}\right) \delta_{G}=\left(\delta_{G} \otimes i\right) \delta_{G} .
$$

$\delta_{G}$ is called a comultiplication since we can define a multiplication on $A(G)=\mathscr{L}(G)_{*}=$ the predual of $\mathcal{L}(G)$ (this is usually called the Fourieralgebra of $G)$ by

$$
\langle a, \alpha \beta\rangle=\left\langle\delta_{G}(a), \alpha \otimes \beta\right\rangle \text { for } a \in \mathcal{L}(G), \alpha, \beta \in A(G) .
$$


It is known that $A(G)$ can be considered a subspace of $C_{0}(G)$, and the multiplication defined by (0.6) coincides with pointwise multiplication of functions in $C_{0}(G)$. The duality theorem for $\mathscr{L}(G)$ then simply says that $G$ is isomorphic to the set of all nonzero elements $x$ of $\mathscr{L}(G)$ satisfying $\delta_{G}(x)=x$ $\otimes x$. (An element in $G$ is identified with its image in $\mathcal{L}(G)$ and $\mathcal{L}(G)$ is given the $\sigma$-topology.) We are not going to use this duality theorem for groups, but it has served as a motivation in formulating our duality theorem for covariance algebras over nonabelian groups which we now shall describe. Suppose $\mathfrak{A}$ is the regular covariance algebra of a covariant system $(A, \rho, G)$ acting on the Hilbert space $X=L^{2}(G, H)$ as described above. Define a unitary operator $W$ over $X \otimes L^{2}(G) \cong L^{2}(G \times G, H)$ by

$$
W f(s, t)=f(s, s t) \text { for } f \in L^{2}(G \times G, H), s, t \in G .
$$

We can then define a ${ }^{*}$-isomorphism $\delta: \mathfrak{A} \rightarrow \mathfrak{A} \otimes \mathfrak{L}(G)$ by

$$
\delta(a)=W^{*}(a \otimes I) W \quad \text { for } a \in \mathfrak{A} .
$$

$\delta$ will then satisfy

$$
\begin{gathered}
\delta(\lambda(x))=\lambda(x) \otimes x, \\
(\delta \otimes i) \delta=\left(i \otimes \delta_{G}\right) \delta,
\end{gathered}
$$

where $i$ denotes the identity map on $\mathscr{L}(G)$ or $\mathfrak{A}$. The duality theorem for covariance algebras over a not necessarily abelian group $G$ (Theorem 1) then says that a von Neumann algebra $\mathfrak{A}$ is isomorphic to the regular covariance algebra of a covariant system $(A, \rho, G)$ over $G$ if and only if there is a normal ${ }^{*}$-isomorphism $\delta$ of $\mathfrak{A}$ into $\mathfrak{A} \otimes \mathfrak{L}(G)$ and a continuous homomorphism $\lambda$ of $G$ into $\mathfrak{A}$ such that $(0.8)$ and $(0.9)$ hold. $A$ and $\rho$ are uniquely determined (up to isomorphisms) by $A=\{a \in \mathfrak{U} \mid \delta(a)=a \otimes I\}$ and $\rho_{x}(a)=\lambda(x) a \lambda\left(x^{-1}\right)$ for $x \in G, a \in A$.

We just saw that the comultiplication $\delta_{G}$ of $\mathscr{L}(G)$ defined a multiplication in $A(G)$, the predual of $\mathcal{L}(G)$. In a similar way a map $\delta$ satisfying (0.9) will define an action of $A(G)$ on the predual $\mathfrak{A}_{*}$ of $\mathfrak{A}$ by

$$
\langle a, \varphi \alpha\rangle=\langle\delta(a), \varphi \otimes \alpha\rangle \quad \text { for } a \in \mathfrak{A}, \varphi \in \mathfrak{A}_{*}, \alpha \in A(G) .
$$

So $\mathfrak{A}_{*}$ is an $A(G)$-module, and accordingly $(\mathfrak{A}, \delta)$ is called a $\mathfrak{L}(G)$-comodule. This is consistent with the terminology used in algebra, cf. [21]. So this describes what could be called the von Neumann algebra version of the duality theorem for regular covariance algebras which appears in Chapter 2.

Let us now sketch how the proof for abelian groups can be extended to the nonabelian case. We see that we need an analogue of the map $P$ defined above and we shall briefly describe how this can be done when $G$ is discrete.

So we shall suppose that we have given a von Neumann algebra $\mathfrak{A}$, a homomorphism $\lambda: G \rightarrow \mathfrak{A}$ and an isomorphism $\delta: \mathfrak{A} \rightarrow \mathfrak{U} \otimes \mathfrak{L}(G)$ satisfying 
$(\delta \otimes i) \delta=\left(i \otimes \delta_{G}\right) \delta$ and $\delta \circ \lambda(x)=\lambda(x) \otimes x$ for $x \in G$. $\mathcal{L}(G)$ has a finite trace tr defined by $\operatorname{tr}(a)=\left\langle a \varepsilon_{e}, \varepsilon_{e}\right\rangle$ for $a \in \mathcal{L}(G)$ where $\varepsilon_{e} \in L^{2}(G)$ is defined by $\varepsilon_{e}(x)=0$ for $x \neq e$ and $\varepsilon_{e}(e)=1$. We can define a normal, bounded, positive linear map $P: \mathfrak{A} \otimes \mathfrak{L}(G) \rightarrow \mathfrak{A}$ such that $P(a \otimes b)=\operatorname{tr}(b) a$ for $a \in \mathfrak{A}, b \in \mathfrak{L}(G)$. Then $P \circ \delta$ will map $\mathfrak{A}$ onto

$$
A=\{a \in \mathfrak{A} \mid \delta(a)=a \otimes I\},
$$

and $P \circ \delta$ turns out to be the analogue of the map $a \rightarrow \int \tau_{\gamma}(a) d \gamma$ defined for an abelian $G$. We can then prove that $A$ and $\lambda(G)$ generates $\mathfrak{A}$ and that $\mathfrak{A}$ is isomorphic to the covariance algebra of $(A, \rho, G)$ where

$$
\rho_{x}(a)=\lambda(x) a \lambda\left(x^{-1}\right) \text { for } a \in A, x \in G .
$$

It is now clear how we should proceed from discrete, nonabelian groups to the general situation. We will have a map $\operatorname{tr}$ of $\mathcal{L}(G)$ also for $G$ nondiscrete, however it is only densely defined, so the corresponding map $P$ is also only densely defined. By carefully using the continuity properties of $P$ developed in Chapter 1 we can prove the duality theorem also for general locally compact groups.

Chapter 3 contains the duality theorem for $C^{*}$-algebras and let us now try to describe this. If $(A, \rho, G)$ is a covariant system with $A$ a $C^{*}$-algebra over a Hilbert space $H$ and $\rho$ is norm-continuous (i.e. $x \rightarrow \rho_{x}(a)$ is norm-continuous for all $a \in A$ ) the regular $C^{*}$-covariance algebra $\mathfrak{A}$ is defined as the $C^{*}$ algebra generated over $L^{2}(G, H)$ by the operators $\tilde{\rho}(\varphi)$ with $\varphi \in C_{00}(G, A)=$ the norm-continuous $A$-valued functions on $G$ with compact support, and $\tilde{\rho}(\varphi)$ is defined by

$$
\tilde{\rho}(\varphi) f(x)=\int \rho_{x^{-1}}(\varphi(y)) f\left(y^{-1} x\right) d y \quad \text { for } f \in L^{2}(G, H), x \in G .
$$

As before we can define a continuous representation $\lambda$ of $G$ by (0.2), but now $\lambda(x)$ is not in $\mathscr{U}$ (this is only the case if $G$ is discrete and $A$ has an identity). However, $\lambda(x) \in M(\mathfrak{U})$, the multiplier algebra of $\mathfrak{A}$, and $\lambda$ is continuous with respect to the multiplier topology on $M(\mathfrak{A})$, i.e. if $x_{i} \rightarrow x$ in $G$ then $\lambda\left(x_{i}\right) a \rightarrow$ $\lambda(x) a$ and $a \lambda\left(x_{i}\right) \rightarrow a \lambda(x)$ in norm for all $a \in \mathfrak{A}$.

Let us describe the situation with $G$ abelian first. (0.3) will then define a norm-continuous homomorphism $\tau: \hat{G} \rightarrow \operatorname{Aut}(\mathfrak{H})$ satisfying (0.4). (Note that a ${ }^{*}$-automorphism of a $C^{*}$-algebra always extends to a ${ }^{*}$-automorphism of its multiplier algebra.) The duality theorem for $C^{*}$-covariance algebras over an abelian group $G$ (Theorem 4 ) now reads as follows: A $C^{*}$-algebra $\mathfrak{A}$ is isomorphic to a $C^{*}$-covariance algebra for some covariant system $(A, \rho, G)$ over $G$ if and only if there is a continuous homomorphism $\lambda: G \rightarrow M(\mathfrak{U})$ and a norm-continuous homomorphism $\tau: \hat{G} \rightarrow \operatorname{Aut}(\mathfrak{A})$ satisfying (0.4). $A$ and $\rho$ are uniquely determined up to isomorphisms by $\rho_{x}(a)=\lambda(x) a \lambda\left(x^{-1}\right)$ and $A$ consists of all elements $a \in M(\mathfrak{H})$ with $\tau_{\gamma}(a)=a$ for all $\gamma \in \hat{G}, a \lambda(f)$ and 
$\lambda(f) a$ are in $\mathfrak{U}$ for all $f \in C_{00}(G)$ and $x \rightarrow \rho_{x}(a)$ is norm-continuous. So we see it is more difficult to find $A$ in this case, the reason being that $A \cap \mathfrak{U} \neq$ $\{0\}$ only if $G$ is discrete, and that $A$ in general may not have an identity.

Let us sketch the proof also in this situation. So suppose we have a $C^{*}$-algebra $\mathfrak{A}$ over a Hilbert space $H$, a continuous homomorphism $\lambda: G \rightarrow$ $M(\mathfrak{U})$ and a norm-continuous homomorphism $\tau: \hat{G} \rightarrow \operatorname{Aut}(\mathfrak{U})$ such that $\tau_{\gamma}(\lambda(x))=\gamma(x) \lambda(x)$ for $x \in G, \gamma \in \hat{G}$. Let $\rho_{x}(a)=\lambda(x) a \lambda\left(x^{-1}\right)$ and let $A=\left\{a \in M(\mathfrak{U}) \mid \tau_{\gamma}(a)=a\right.$ for all $\gamma \in \hat{G}, a \lambda(f)$ and $\lambda(f) a \in \mathfrak{U}$ for all $f \in$ $C_{00}(G)$ and $x \rightarrow \rho_{x}(a)$ is norm-continuous $\}$. Then $A$ is a $C^{*}$-algebra and $\rho_{x} \in \operatorname{Aut}(A)$.

The proof now follows the same line as sketched for the von Neumann algebra situation, but $\sigma$-convergence of course has to be replaced by normconvergence. Define $\mathfrak{A}_{0}, \mathfrak{A}_{1}$ and the map $P$ as above:

$$
\langle P(a) \xi, \eta\rangle=\int_{\hat{G}}\left\langle\tau_{\gamma}(a) \xi, \eta\right\rangle d \gamma \text { for } a \in \mathfrak{A}_{1} .
$$

It turns out that $P(a)$ is not necessarily in $\mathfrak{U}$, not even in $M(\mathfrak{U})$ for $a \in \mathfrak{A}_{1}$, but we can prove that if $f, g \in C_{00}(G), a \in \mathfrak{A}$, then $P\left(\lambda(g)^{*} a \lambda(f)\right) \in A$. Now take $b=\lambda(g)^{*} a \lambda(f)$ (note that elements of this form are norm-dense in $\mathfrak{A}$ ) and take a net $\left\{\varphi_{i}\right\}$ from $L^{1}(G)$ such that the net $\left\{\hat{\varphi}_{i}\right\}$ of Fourier-transforms is an approximate identity in $L^{1}(\hat{G})$. With $b_{i}=\int_{G} \varphi_{i}(x) P(b \lambda(x)) \lambda\left(x^{-1}\right) d x$ we will have that $b_{i} \rightarrow b$ in norm. This is the essential part of the proof, it is not difficult now to conclude that $\mathfrak{A}$ is isomorphic to the $C^{*}$-covariance algebra of $(A, \rho, G)$.

In order to extend this theorem to $C^{*}$-covariance algebras over a nonabelian group $G$ we have already done much of the ground work when describing the duality theorem for von Neumann covariance algebras. We of course want to replace $\mathcal{L}(G)$ with $C_{r}^{*}(G)$ (the $C^{*}$-algebra generated by the left regular representation of $\left.L^{1}(G)\right)$ and define the maps $\delta_{G}$ and $\delta$ by $(0.5)$ and (0.7). The first problem arising is that we usually will have $\delta(\mathfrak{A}) \& \mathfrak{A} \otimes$ $C_{r}^{*}(G)$ and similarly for $\delta_{G}\left(C_{r}^{*}(G)\right)$, but we will have $\delta(\mathfrak{A}) \subset M(\mathfrak{A} \otimes$ $C_{r}^{*}(G)$ ). For the general duality theorem for $C^{*}$-covariance algebras (Theorem 3), see Chapter 3. It should be noted that our duality theorems are always stated with respect to some fixed group $G$, we do not answer the question when an operator algebra $\mathfrak{A}$ is the covariance algebra of some covariant system $(A, \rho, G)$ over some group $G$. Of course this problem always will have the trivial solution obtained by taking $G=\{e\}$ and $A=\mathfrak{A}$. Furthermore, this problem cannot have a unique solution in general. For instance if we have a covariant system $(A, \rho, G)$ where the group $G$ is a semidirect product of a closed normal subgroup $N$ and a closed subgroup $H$, then the covariance algebra $\mathfrak{A}$ of $(A, \rho, G)$ will also be the covariance algebra of another covariant system $(\mathfrak{B}, \tilde{\rho}, H)$. Here $\mathfrak{B}$ is the covariance algebra of 
$(A, \rho \mid N, N)$ and $\tilde{\rho}: H \rightarrow \operatorname{Aut}(\mathfrak{B})$ is a natural "extension" of $\rho$ to $\mathfrak{B}$.

However, some work in this direction has been done by J. Ernest in [14]. If $\mathfrak{U}$ is the von Neumann regular covariance algebra of $(A, \rho, G)$ we have seen how we can define the comodule map $\delta: \mathfrak{A} \rightarrow \mathfrak{U} \otimes \mathfrak{L}(G)$. The map $\lambda: G \rightarrow \mathfrak{U}$ extends to a normal *-isomorphism of $\mathscr{L}(G)$ into $\mathfrak{A}$ and the map $d=(i \otimes \lambda) \delta: \mathfrak{A} \rightarrow \mathfrak{A} \otimes \mathfrak{A}$ is then a comultiplication of $\mathfrak{A}$ (i.e. $(i \otimes d) d=$ $(d \otimes i) d)$. Ernest then proves that $G$ is algebraically and topologically isomorphic to the intrinsic group $G(\mathfrak{A}, d)$ of the Hopf-von Neumann algebra $(\mathfrak{A}, d)$. Here $G(\mathfrak{A}, d)=\{a \in \mathfrak{A} \mid a$ is invertible and $d(a)=a \otimes a\}$ with the $\sigma$-topology. It would be interesting to know under what conditions a given Hopf-von Neumann algebra $(\mathfrak{A}, d)$ is the covariance algebra for some covariant system over its intrinsic group $G(\mathfrak{A}, d)$.

This paper forms the major portion of the author's Doctoral Dissertation written at the University of Pennsylvania and I gratefully acknowledge the financial support given me by The Norwegian Research Council for Science and the Humanities (NAVF) and the University of Pennsylvania. I also want to thank my adviser Professor J.M.G. Fell for his moral and mathematical support and U. Haagerup who together with J.M.G. Fell pointed out several errors in an earlier version of this work.

Notation. The terminology and notation will mostly agree with the one in the books of J. Dixmier and S. Sakai ([8], [9] and [20]).

If $A$ is a $C^{*}$-algebra, $A^{+}$denotes all positive elements in $A, A^{*}$ is the norm-dual of $A, A^{*+}$ consists of all positive functionals in $A^{*}$ and

$$
S(A)=\left\{\varphi \in A^{*+} \mid\|\psi\|=1\right\}=\text { the state-space of } A .
$$

$\operatorname{Aut}(A)$ is the group of all *-automorphisms of $A$, and if $A$ has an identity $A_{u}$ is the group of unitary elements in $A$.

If $A$ is a von Neumann algebra then $A_{*}=$ the predual of $A=$ all normal linear functionals on $A, A_{*}^{+}=$all positive functionals in $A_{*}$ and the $\sigma$ topology on $A$ is defined by

$$
a_{i} \rightarrow a \Leftrightarrow \varphi\left(a_{i}\right) \rightarrow \varphi(a) \text { for all } \varphi \in A_{*} .
$$

For the duality between $A$ and $A^{*}$ or $A_{*}$ we will use one of the identical expressions

$$
\langle a, \varphi\rangle \text { or } \varphi(a), \quad a \in A, \varphi \in A^{*} .
$$

If $X$ is a Hilbert space, $\mathscr{B}(X)=$ all bounded linear operators on $X$ and $C C(X)=$ all compact operators in $\mathscr{B}(X)$. If $C$ is a subset of $\mathscr{B}(X)$, its commutant $C^{\prime}$ and double commutant $C^{\prime \prime}=\left(C^{\prime}\right)^{\prime}$ is defined as usual. If $A$ is a $C^{*}$-algebra, its second dual $A^{* *}$ is in a natural way a von Neumann algebra-in fact if $\pi$ is the universal representation of $A$, we have that $A^{* *} \cong \pi(A)^{\prime \prime}$, cf. $[20,1.17 .2]$ or $[9,12.1]$. 
If $G$ is a locally compact group, the spaces $L^{p}(G), 1 \leqslant p \leqslant \infty$, are defined with respect to a left invariant Haar-measure on $G$. If $H$ is a Hilbert space, $L^{2}(G, H)$ is the Hilbert space of (equivalence classes of) measurable functions $f: G \rightarrow H$ with $\|f\|_{2}=\left(\int_{G}\|f(x)\|^{2} d x\right)^{1 / 2}<\infty$, this space is isomorphic to the Hilbert space tensor product $H \otimes L^{2}(G)$. If $\xi \in H, f \in$ $L^{2}(G), \xi \otimes f \in L^{2}(G, H)$ is defined by $(\xi \otimes f)(x)=f(x) \xi$ for $x \in G$.

If $X$ is a locally compact Hausdorff space and $E$ is a Banach space, $C B(X, E)=$ all bounded, continuous functions from $X$ to $E, C_{00}(X, E)=$ all functions in $C B(X, E)$ vanishing outside a compact set and $C_{0}(X, E)=$ all functions in $C B(X, E)$ vanishing at infinity. If $E=\mathbf{C}=$ the field of complex numbers, we simply write $C B(X), C_{00}(X)$ and $C_{0}(X)$. If $A$ is a von Neumann algebra and $(X, \mu)$ is a measure space, then $L^{\infty}(X, A)$ is the von Neumann algebra of (equivalence classes of) all essentially bounded measurable functions from $X$ to $A$.

The tensor product of two operator algebras $A$ and $B$ over Hilbert spaces $X$ and $Y$ respectively is defined as follows: If $A$ and $B$ are $C^{*}$-algebras, $A \otimes B$ is the $C^{*}$-algebra generated by the algebraic tensor product $A \otimes_{\text {alg }} B$ over the Hilbert space $X \otimes Y$. If $A$ and $B$ are von Neumann algebras, $A \otimes B$ will be the von Neumann algebra generated by $A \otimes_{\text {alg }} B$. It turns out that these definitions do not depend on the Hilbert spaces $X$ and $Y$ on which we represent $A$, respectively $B$, cf. [12].

If $A$ is a $C^{*}$-algebra over a Hilbert space $X$, let $M(A)$, the multiplier algebra or double centralizer algebra of $A$, be defined by $M(A)=\{a \in$ $\left.A^{\prime \prime} \mid a A+A a \subseteq A\right\}$. This definition does not depend on $X$, cf. [3]. The multiplier-topology on $M(A)$ is defined by:

$$
m_{i} \rightarrow m \Leftrightarrow\left\|a m_{i}-a m\right\|+\left\|m_{i} b-m b\right\| \rightarrow 0 \text { for all } a, b \in A .
$$

$A$ is dense in $M(A)$ in this topology. It is important to note that in general $M(A) \otimes M(B) \subsetneq M(A \otimes B)$ for two $C^{*}$-algebras $A$ and $B$, cf. [1]. We shall also need the following object:

$$
\tilde{M}(A \otimes B)=\{x \in M(A \otimes B) \mid x(I \otimes B)+(I \otimes B) x \subseteq A \otimes B\} .
$$

If $B$ is an abelian $C^{*}$-algebra, we have the following characterization of $\tilde{M}(A \otimes B)$.

LeMmA 0.1. If $X$ is a locally compact Hausdorff space, then $\tilde{M}\left(A \otimes C_{0}(X)\right)$ $\cong C B(X, A)$.

Proof. By [20, 1.22.3] and [1] we have that $A \otimes C_{0}(X) \cong C_{0}(X, A)$ and $M\left(C_{0}(X, A)\right) \cong C B(X, M(A))$.

If $f \in C B(X, M(A))$ is in $\tilde{M}\left(A \otimes C_{0}(X)\right)$ then $\varphi f \in C_{0}(X, A)$ for all $\varphi \in C_{0}(X)$, where $\varphi f$ is defined by $\varphi f(x)=\varphi(x) f(x)$. So $f$ takes values in $A$, i.e. $f \in C B(X, A)$. Conversely if $f \in C B(X, A)$ and $\varphi \in C_{0}(X)$, then $\varphi f \in$ $C_{0}(X, A)$, so $f \in \tilde{M}\left(A \otimes C_{0}(X)\right)$. Hence $\tilde{M}\left(A \otimes C_{0}(X)\right) \cong C B(X, A)$. 


\section{Chapter 1. Tensor Products of Weights and}

\section{NONCOMMUTATIVE INTEGRATION THEORY}

If $\mathfrak{A}$ and $\mathfrak{B}$ are $C^{*}$-algebras and $\varphi \in \mathfrak{B}^{*}$ we can define a map $P_{\varphi}$ from $\mathfrak{A} \otimes \mathfrak{B}$ to $\mathfrak{A}$ by the formula

$$
\left\langle P_{\varphi}(x), \psi\right\rangle=\langle x, \psi \otimes \varphi\rangle \quad \text { for } x \in \mathfrak{A} \otimes \mathfrak{B}, \psi \in \mathfrak{U}^{*} .
$$

This map was studied by J. Tomiyama in [29]. In this chapter we shall study the map $P_{\varphi}$ obtained by instead letting $\varphi$ be a weight on $\mathfrak{B}$. The unboundedness of $\varphi$ will imply that $P_{\varphi}$ is not everywhere defined, and we shall study the continuity properties of $P_{\varphi}$ still remaining.

First we shall review some results on weights on operator algebras and left generalized Hilbert algebras. As references we shall use [16, §2], [4], [5] or [24].

If $\mathfrak{A}$ is a $C^{*}$-algebra, a map $\varphi: \mathfrak{A}^{+} \rightarrow[0, \infty]$ is called a weight on $\mathfrak{U}$ if

$$
\begin{gathered}
\varphi(x+y)=\varphi(x)+\varphi(y) \text { for } x, y \in \mathfrak{A}^{+}, \\
\varphi(\lambda x)=\lambda \varphi(x) \quad \text { for } x \in \mathfrak{U}^{+}, \lambda \geqslant 0
\end{gathered}
$$

(with the convention that $0 \cdot \infty=0$ ).

Let $\mathfrak{U}_{\varphi}^{+}=\left\{a \in \mathfrak{U}^{+} \mid \varphi(a)<\infty\right\} . \widetilde{\Im}_{\varphi}=\left\{a \in \mathfrak{A} \mid a^{*} a \in \mathfrak{A}_{\varphi}^{+}\right\}$is then a left ideal in $\mathfrak{A}, \mathfrak{A}_{\varphi}=\mathfrak{J}_{\varphi}^{*} \mathfrak{J}_{\varphi}=$ linspan $\mathfrak{A}_{\varphi}^{+}$is a ${ }^{*}$-subalgebra of $\mathfrak{A}$ and $\varphi$ extends uniquely to a positive linear functional on $\mathfrak{A}_{\varphi}$.

If $\mathfrak{U}$ is a von Neumann algebra, $\varphi$ is called normal if there is a set $\left\{\varphi_{i}\right\}$ of (bounded) normal, positive functionals on $\mathfrak{A}$ such that $\varphi(x)=\sup \varphi_{i}(x)$ for all $x \in \mathfrak{A}^{+} . \varphi$ is said to be faithful if $\varphi\left(x^{*} x\right)=0 \Rightarrow x=0$, and if $\mathfrak{U}_{\varphi}$ is $\sigma$-dense, $\varphi$ is said to be semifinite. We shall call $\varphi$ lower semicontinuous if $\varphi$ is lower semicontinuous on $\mathfrak{A}^{+}$in the $\sigma$-topology. It then follows from [4, Proposition 1.7 and Lemma 4.3] that there is an increasing net $\left\{\varphi_{i}\right\}$ from $\mathfrak{A}_{*}^{+}$ such that

$$
\varphi(a)=\sup _{i} \varphi_{i}(a) \quad \text { for } a \in \mathfrak{A}_{\varphi}^{+} .
$$

$A$ left generalized Hilbert algebra is a ${ }^{*}$-algebra $A$ equipped with a positive, Hermitian form $\langle$,$\rangle such that$

$$
\langle a b, c\rangle=\left\langle b, a^{*} c\right\rangle \quad \text { for } a, b, c \in A .
$$

For $a \in A$ there is $K \geqslant 0$ such that $\langle a b, a b\rangle \leqslant K\langle b, b\rangle$ for all $b \in A$.

The subalgebra $A^{2}$ is dense in $A$ with respect to the pseudonorm defined by $\langle$,$\rangle .$ 
Let $H$ be the Hilbert space completion of $A$ with respect to (iv) $\langle$,$\rangle .Then there shall be a closed operator from H$ into the conjugate Hilbert space $\bar{H}$ which extends the map $a \rightarrow a^{*}$ from $A$ to $A$.

Let $\sim$ be the canonical map from $A$ into $H$, then there is a natural nondegenerate ${ }^{*}$-representation $\pi$ of $A$ over $H$ such that $\pi(a) \tilde{b}=(a b)^{\sim}$ for $a$, $b \in A$.

A vector $\alpha \in H$ is called right bounded if the map $a \rightarrow \pi(a) \alpha$ from $A$ to $H$ is bounded. Let $H_{r}$ be the linear space of all such vectors, so any $\alpha \in H_{r}$ will define a bounded linear operator $\pi^{\prime}(\alpha)$ over $H$ such that $\pi^{\prime}(\alpha) \tilde{a}=\pi(a) \alpha$ for $a \in A$, and $\pi^{\prime}(\alpha) \in \pi(A)^{\prime} . \alpha \in H$ is called left bounded if the map $a \rightarrow \pi^{\prime}(a) \alpha$ from $H_{r}$ to $H$ is bounded. If $H_{l}$ is the linear space of all such vectors, we have $\tilde{A} \subset H_{l}$ and each $\alpha \in H_{l}$ defines an operator $\pi(\alpha)$ satisfying $\pi(\alpha) a=$ $\pi^{\prime}(a) \alpha$ for $a \in H_{r}$. Let $\mathfrak{A}=\pi(A)^{\prime \prime}$, then $\pi\left(H_{l}\right) \subset \mathfrak{A}$ and we can define a faithful, normal, semifinite and lower semicontinuous weight $\varphi$ on $\mathfrak{A}^{+}$as follows, cf. [5, Théorème 2.11]: If $a \in \mathfrak{A}^{+}$and $a=\pi(\alpha)^{*} \pi(\alpha)$ for some $\alpha \in H_{l}$ define $\varphi(a)=\langle\alpha, \alpha\rangle$, if no such $\alpha$ exists take $\varphi(a)=\infty$.

Conversely, if $\varphi$ is a faithful, normal and semifinite weight on a von Neumann algebra $\mathfrak{A}$, then $A=\widetilde{\mho}_{\varphi} \cap \mathfrak{\mho}_{\varphi}^{*}$ is a left generalized Hilbert algebra if we define $\langle a, b\rangle=\varphi\left(b^{*} a\right)$ for $a, b \in A$. Let $H$ be the corresponding Hilbert space as defined above, and let $\mathfrak{U}_{\varphi}^{\prime}=\left\{a \in A \mid \tilde{a} \in H_{r}\right\}$. It follows from the proof of Lemma 3.3 in [24] that $\mathfrak{A}_{\varphi}^{\prime}$ is dense in $H$.

Suppose now we have two von Neumann algebras $\mathfrak{A}$ and $\mathfrak{B}$ each having normal, semifinite weights $\varphi$ and $\omega$ respectively. Let $\left\{\varphi_{i}\right\}$ be a net from $\mathfrak{A}_{*}^{+}$ and $\left\{\omega_{j}\right\}$ a net from $\mathfrak{B}_{*}^{+}$such that

$$
\begin{array}{ll}
\varphi(a)=\sup _{i} \varphi_{i}(a) & \text { for } a \in \mathfrak{U}^{+}, \\
\omega(a)=\sup _{j} \omega_{j}(b) & \text { for } b \in \mathfrak{B}^{+} .
\end{array}
$$

Let $\mathfrak{A} \otimes \mathfrak{B}$ be the von Neumann algebra tensor product as defined earlier. For each $i, j$ we can define $\varphi_{i} \otimes \omega_{j} \in(\mathfrak{A} \otimes \mathfrak{B})^{+}$such that

$$
\left(\varphi_{i} \otimes \omega_{j}\right)(a \otimes b)=\varphi_{i}(a) \omega_{j}(b) \quad \text { for } a \in \mathfrak{U}, b \in \mathfrak{B} .
$$

Then define for $x \in(\mathfrak{A} \otimes \mathfrak{B})^{+}$,

$$
\varphi \otimes \omega(x)=\sup _{i, j} \varphi_{i} \otimes \omega_{j}(x) .
$$

$\varphi \otimes \omega$ will then be a normal semifinite weight on $\mathfrak{A} \otimes \mathfrak{B}$ satisfying

$$
(\varphi \otimes \omega)(a \otimes b)=\varphi(a) \omega(b) \quad \text { for } a \in \mathfrak{A}^{+}, b \in \mathfrak{B}^{+} .
$$

Let $(\mathfrak{A} \otimes \mathfrak{B})_{\omega}^{+}$be the set of all $x \in(\mathfrak{A} \otimes \mathfrak{B})^{+}$satisfying

$$
\exists M \geqslant 0 \text { such that } \varphi \otimes \omega(x) \leqslant M \text { for all } \varphi \in S(\mathfrak{H})
$$


where $S(\mathfrak{U})$ is the set of all normal states of $\mathfrak{U}$. Let $\mathfrak{\mho}_{\omega}$ consist of all elements $x$ from $\mathfrak{U} \otimes \mathfrak{B}$ with $x^{*} x \in(\mathfrak{A} \otimes \mathfrak{B})_{\omega}^{+}$and let $(\mathfrak{A} \otimes \mathfrak{B})_{\omega}=\operatorname{linspan}(\mathfrak{H} \otimes \mathfrak{B})_{\omega}^{+}$ $=\mathfrak{\mho}_{\omega}^{*} \mathfrak{\mho}_{\omega}$.

We can now define a map $P_{\omega}$ from $(\mathfrak{A} \otimes \mathfrak{B})_{\omega}$ into $\mathfrak{U}$ by the requirement

$$
\left\langle P_{\omega}(x), \varphi\right\rangle=\langle x, \varphi \otimes \omega\rangle \quad \text { for all } \varphi \in \mathfrak{U}_{*} .
$$

$P_{\omega}$ is obviously linear and is positive, i.e. $x \in(\mathfrak{A} \otimes \mathfrak{B})_{\omega}^{+} \Rightarrow P_{\omega}(x) \in \mathfrak{A}^{+}$. Furthermore, if $x \in(\mathfrak{A} \otimes \mathfrak{B})_{\omega}$ and $a, b \in \mathfrak{U}$ then

$$
(a \otimes I) x(b \otimes I) \in(\mathfrak{A} \otimes \mathfrak{B})_{\omega} \text { and } P_{\omega}((a \otimes I) x(b \otimes I))=a P_{\omega}(x) b .
$$

If $a \in \mathfrak{A}$ and $b \in \mathfrak{B}_{\omega}, a \otimes b \in(\mathfrak{A} \otimes \mathfrak{B})_{\omega}$ and $P_{\omega}(a \otimes b)=\omega(b) a$.

If $\omega$ is bounded, $(\mathfrak{A} \otimes \mathfrak{B})_{\omega}=\mathfrak{A} \otimes \mathfrak{B}$ and the map $P_{\omega}$ will be a bounded, positive linear map onto $\mathfrak{A}$. This case has been studied in [29].

We shall now study the continuity properties of the map $P_{\omega}$. Through the rest of this chapter $\omega$ shall be some fixed faithful normal semifinite lower semicontinuous weight on $\mathfrak{B}$, and we shall simply write $P$ for $P_{\omega}$. Convergence is always with respect to the $\sigma$-topology.

LeMma 1.1. $P$ is normal, i.e. if $\left(a_{i}\right)_{i \in I}$ is an increasing net in $(\mathfrak{A} \otimes \mathfrak{B})^{+}$ converging to $a \in(\mathfrak{A} \otimes \mathfrak{B})_{\omega}^{+}$in the $\sigma$-topology, then $P\left(a_{i}\right) \rightarrow P(a)$.

Proof. If $\varphi \in \mathfrak{A}_{*}^{+}$and $\varepsilon>0$ are given, there is a $\psi \in(\mathfrak{A} \otimes \mathfrak{B})_{*}^{+}$with $\psi \leqslant \varphi \otimes \omega$ and $\varphi \otimes \omega(a)-\psi(a)<\varepsilon / 2$. For some $i_{0} \in I$ we will have that $\psi\left(a-a_{i}\right)<\varepsilon / 2$ for all $i>i_{0}$. So for $i>i_{0}$

$$
\begin{aligned}
0 \leqslant & \varphi\left(P\left(a-a_{i}\right)\right)=\varphi(P(a))-\psi(a)+\psi(a)-\psi\left(a_{i}\right) \\
& +\psi\left(a_{i}\right)-\varphi\left(P\left(a_{i}\right)\right)<(\varphi \otimes \omega-\psi)(a)+\varepsilon / 2 \\
& +(\psi-\varphi \otimes \omega)\left(a_{i}\right)<\varepsilon / 2+\varepsilon / 2+0=\varepsilon .
\end{aligned}
$$

Thus $P\left(a_{i}\right) \rightarrow P(a)$ in the $\sigma$-topology.

LEMMA 1.2. Let $b$ be a positive element of a von Neumann algebra $\mathfrak{A}, p$ the range-projection of $b$. The map $\Phi$ defined by $\Phi(a)=b^{1 / 2} a b^{1 / 2}$ is then $a$ homeomorphism between

and

$$
C=\{a \in \mathfrak{A} \mid-p \leqslant a \leqslant p\}
$$

$$
D=\{a \in A \mid-b \leqslant a \leqslant b\}
$$

with respect to the $\sigma$-topology.

Proof. $\Phi$ is obviously $\sigma$-continuous, $C$ is $\sigma$-closed and norm-bounded, so $C$ is compact in the $\sigma$-topology. Therefore it suffices to prove that $\Phi$ is a bijection between $C$ and $D$. 
To prove that $\Phi$ is one-to-one it suffices to prove that $a \in C, \Phi(a)=0 \Rightarrow a$ $=0$. If $b^{1 / 2} a b^{1 / 2}=0$ then also pap $=0$. Since $0 \leqslant a+p \leqslant 2 p$ we have that

$$
\begin{aligned}
p \xi=0 & \Rightarrow\left\|(a+p)^{1 / 2} \xi\right\|^{2}=\langle(a+p) \xi, \xi\rangle \leqslant\langle 2 p \xi, \xi\rangle=0 \\
& \Rightarrow(a+p)^{1 / 2} \xi=0 \Rightarrow(a+p) \xi=0 \Rightarrow a \xi=0 .
\end{aligned}
$$

So $\operatorname{ker} p \subset \operatorname{ker} a$, thus range $a \subset$ range $p$ so $p a=a p=a$. Hence $a=p a p=$ 0 .

To prove that $\Phi$ is onto, suppose $d \in D$ is given. Then

$$
\begin{aligned}
\left.\langle(d+b) \xi, \eta\rangle\right|^{2} & \leqslant\langle(d+b) \xi, \xi\rangle\langle(d+b) \eta, \eta\rangle \\
& \leqslant\langle 2 b \xi, \xi\rangle\langle 2 b \eta, \eta\rangle \leqslant 4\left\|b^{1 / 2} \xi\right\|^{2}\left\|b^{1 / 2} \eta\right\|^{2}
\end{aligned}
$$

so there is an element $c \in \mathfrak{U}$ with $p c p=c$ such that $\left\langle c b^{1 / 2} \xi, b^{1 / 2} \eta\right\rangle=\langle(d+$ $b) \xi, \eta>$ for all $\xi, \eta$.

We have $\left\langle c b^{1 / 2} \xi, b^{1 / 2} \xi\right\rangle=\langle(d+b) \xi, \xi\rangle \leqslant 2\left\langle p b^{1 / 2} \xi, b^{1 / 2} \xi\right\rangle$. So $0 \leqslant c \leqslant$ $2 p$, and $a=c-p \in C$ satisfies

$$
\Phi(a)=b^{1 / 2} a b^{1 / 2}=b^{1 / 2} c b^{1 / 2}-b=d+b-b=d .
$$

So $\Phi$ is onto.

CoRollary 1.3. If $b, c \in \mathfrak{\Im}_{\omega}$ the map $a \rightarrow P\left(b^{*} a c\right)$ from $\mathfrak{A} \otimes \mathfrak{B}$ to $\mathfrak{A}$ is $\sigma$-continuous on bounded spheres. Furthermore if $\left\{a_{i}\right\}$ is a net of selfadjoint elements from $(\mathfrak{A} \otimes \mathfrak{B})_{\omega}$ converging to $a \in(\mathfrak{A} \otimes \mathfrak{B})_{\omega}$ and if there is $a b \in$ $(\mathfrak{U} \otimes \mathfrak{B})_{\omega}^{+}$with $-b \leqslant a_{i} \leqslant b$ for all $i$, then $P\left(a_{i}\right) \rightarrow P(a)$.

Proof. For the first part it suffices to prove that $a \rightarrow P\left(b^{*} a b\right)$ is $\sigma$ continuous for all $b \in \mathfrak{\Im}_{\omega}$, i.e. that $a \rightarrow \varphi\left(P\left(b^{*} a b\right)\right)$ is $\sigma$-continuous for all $\varphi \in \mathfrak{A}_{*}^{+}$. But $\varphi\left(P\left(b^{*} a b\right)\right)=\varphi \otimes \omega\left(b^{*} a b\right)$, so this is obvious. To prove the last part, consider the map $\Phi$ defined in Lemma 1.2. Then $c_{i}=\Phi^{-1}\left(a_{i}\right) \rightarrow c$ $=\Phi^{-1}(a)$. By the first part $P\left(b^{1 / 2} c_{i} b^{1 / 2}\right) \rightarrow P\left(b^{1 / 2} c b^{1 / 2}\right)$, i.e. $P\left(a_{i}\right) \rightarrow P(a)$. The idea of using Lemma 1.2 in this proof is due to U. Haagerup.

LEMma 1.4. If $x \in(\mathfrak{A} \otimes \mathfrak{B})_{\omega}$ and $e_{i} \in \mathfrak{B}_{\omega}^{\prime}$ with $\pi^{\prime}\left(e_{i}\right) \rightarrow I$ in the $\sigma$-topology then

$$
P\left[\left(I \otimes e_{i}^{*}\right) x^{*} x\left(I \otimes e_{i}\right)\right] \rightarrow P\left(x^{*} x\right) .
$$

Proof. Here $\pi^{\prime}\left(e_{i}\right)$ denotes the operator over a Hilbert space $H_{\omega}$ defined earlier. Note that for $a, b \in \mathfrak{B}_{\omega}^{\prime}, \pi^{\prime}(a b)=\pi^{\prime}(b) \pi^{\prime}(a)$.

It will suffice to prove that for all $\varphi \in \mathfrak{A}_{*}^{+}$.

$$
\left\langle P\left[\left(I \otimes e_{i}^{*}\right) x^{*} x\left(I \otimes e_{i}\right)\right], \varphi\right\rangle \rightarrow\left\langle P\left(x^{*} x\right), \varphi\right\rangle
$$

i.e. that

$$
\left\langle\left(I \otimes e_{i}^{*}\right) x^{*} x\left(I \otimes e_{i}\right), \varphi \otimes \omega\right\rangle \rightarrow\left\langle x^{*} x, \varphi \otimes \omega\right\rangle .
$$

Let $H_{\varphi}$ be the Hilbert space on which the representation $\pi_{\varphi}$ corresponding to the positive linear functional $\varphi$ acts, so we have a natural map $\sim: \mathfrak{A} \rightarrow H_{\varphi}$ 
such that $\langle\tilde{a}, \tilde{b}\rangle=\varphi\left(b^{*} a\right)$ for $a, b \in \mathfrak{A}$. (This is the usual GNSconstruction.) We therefore also have a map $\sim:(\mathfrak{A} \otimes \mathfrak{B})_{\omega} \rightarrow H_{\varphi} \otimes H_{\omega}$ such that $\langle\tilde{x}, \tilde{y}\rangle=\left\langle y^{*} x, \varphi \otimes \omega\right\rangle$ for $x, y \in(\mathfrak{U} \otimes \mathfrak{B})_{\omega}$. We now have

$$
\begin{aligned}
\left\langle\left(I \otimes e_{i}^{*}\right) x^{*} x\left(I \otimes e_{i}\right), \varphi \otimes \omega\right\rangle & \\
& =\left\langle\left(\pi_{\varphi} \otimes \pi\right)(x) I \tilde{\otimes} e_{i},\left(\pi_{\varphi} \otimes \pi\right)(x) I \tilde{\otimes} e_{i}\right\rangle \\
& =\left\langle I \otimes \pi^{\prime}\left(e_{i}\right) \tilde{x}, I \otimes \pi^{\prime}\left(e_{i}\right) \tilde{x}\right\rangle \rightarrow\langle\tilde{x}, \tilde{x}\rangle \\
& =\left\langle x^{*} x, \varphi \otimes \omega\right\rangle .
\end{aligned}
$$

Here we have used the fact that for $x \in(\mathfrak{A} \otimes \mathfrak{B})_{\omega}, e \in \mathfrak{B}_{\omega}^{\prime}$

$$
\left(\pi_{\varphi} \otimes \pi\right)(x) I \tilde{\otimes} e=\left(I \otimes \pi^{\prime}(e)\right) \tilde{x} .
$$

(1.3) obviously holds for $x \in \mathfrak{U} \otimes_{\text {alg }} \mathfrak{B}_{\omega}$ and since this set is dense in $(\mathfrak{U} \otimes \mathfrak{B})_{\omega}$ both in the $\sigma$-topology and in the norm-topology from the Hilbert space $H_{\varphi} \otimes H_{\omega},(1.3)$ holds for all $x \in(\mathfrak{A} \otimes \mathfrak{B})_{\omega}$.

As before let $\mathfrak{A}$ and $\mathfrak{B}$ be $C^{*}$-algebras with a weight $\omega$ on $\mathfrak{B}$, but let us drop the assumption that they are von Neumann algebras. We shall suppose that $\omega$ is faithful and lower semicontinuous and that $\mathfrak{B}_{\omega}$ is norm-dense in $\mathfrak{B}$. Then $\omega$ has an extension to a normal semifinite weight on the von Neumann algebra $\mathfrak{B}^{* *}$, cf. [4, Proposition 4.1]. $\mathfrak{B}_{\omega}$ will be $\sigma$-dense in $\mathfrak{B}_{\omega}^{* *}$ and $\mathfrak{B}$ will have an approximate identity $\left\{e_{i}\right\}$ with each $e_{i} \in \mathfrak{B}_{\omega}$. As before we can define the linear map $P=P_{\omega}:\left(\mathfrak{A}^{* *} \otimes \mathfrak{B}^{* *}\right)_{\omega} \rightarrow \mathfrak{U}^{* *}$.

We want to see what $P$ does to elements which also are in $M(\mathfrak{A} \otimes \mathscr{B})$. One might hope that if $a \in\left(\mathfrak{U}^{* *} \otimes \mathfrak{B}^{* *}\right)_{\omega} \cap(\mathfrak{A} \otimes \mathfrak{B})$, then $P(a)$ is in $\mathfrak{A}$ or at least in $M(\mathfrak{A})$, but the following example shows that this is not true: Take $\mathfrak{A}=C B([-1,1]), \mathfrak{B}=C_{0}(\mathbf{R}), \omega$ as the Lebesgue measure on $\mathbf{R}$. Let $f \in \mathfrak{A} \otimes$ $\mathfrak{B} \cong C_{0}([-1,1] \times \mathbf{R})$ be defined by

$$
f(x, y)= \begin{cases}\frac{x}{1+x^{2} y^{2}} & \text { for } 0<x \leqslant 1, \\ 0 & \text { for }-1 \leqslant x \leqslant 0 .\end{cases}
$$

Then

$$
P(f)(x)=\int_{\mathbf{R}} f(x, y) d y= \begin{cases}\pi & \text { for } 0<x \leqslant 1 \\ 0 & \text { for }-1 \leqslant x \leqslant 0 .\end{cases}
$$

So $P(f) \notin M(\mathfrak{U})=C B([-1,1])$. However, we will have the following:

LeMma 1.5. If $c \in \tilde{M}(\mathfrak{A} \otimes \mathfrak{B}) \cap\left(\mathfrak{A}^{* *} \otimes \mathfrak{B}^{* *}\right)_{\omega}$ and $b \in \mathfrak{B}_{\omega}$, then

$$
P(c(I \otimes b)) \in \mathfrak{A} \text {. }
$$

Proof. To prove the lemma it will suffice to show that $P\left(c^{*} c(I \otimes b)\right) \in \mathfrak{A}$ if $b \in \mathfrak{B}_{\omega}$ and $c \in \tilde{M}(\mathfrak{H} \otimes \mathfrak{B})$ with $c^{*} c \in(\mathfrak{U} \otimes \mathfrak{B})_{\omega}^{+}$.

If $\omega$ is bounded, it is easy to see that $P_{\omega}$ is a bounded linear map from 
$\mathfrak{A} \otimes \mathfrak{B}$ to $\mathfrak{A}$, cf. [29]. So for a general weight $\omega$

$$
P\left(\left(I \otimes e^{*}\right) c(I \otimes e)\right) \in \mathfrak{A} \quad \text { for all } c \in \mathfrak{U} \otimes \mathfrak{B}, e \in \mathfrak{B}_{\omega},
$$

thus also $P\left(\left(I \otimes e^{*}\right) c(I \otimes b)\right) \in \mathfrak{A}$ for all $e, b \in \mathfrak{B}_{\omega}$. Now suppose $\psi \in \mathfrak{U}^{*}$, then $\psi$ has a polar decomposition: $\psi(a)=\varphi(a v)$ for all $a \in \mathfrak{A}$, where $\varphi \in$ $\mathfrak{A}^{*+}, v$ is a partial isometry in $\mathfrak{A}^{* *}$ and $\|\psi\|=\|\varphi\|$ (cf. [20, 1.14.4]).

As in the proof of Lemma 1.4 consider the Hilbert space $H_{\varphi}$ and the representation $\pi_{\varphi}$ of $\mathfrak{A}^{* *}$ on $H_{\varphi}$. The Hilbert space $H_{\omega}$, the map

$$
\sim:\left(\mathfrak{U}^{* *} \otimes \mathfrak{B}^{* *}\right)_{\omega} \rightarrow H_{\varphi} \otimes H_{\omega}
$$

and the operators $\pi(a)$ and $\pi^{\prime}(b)$ over $H_{\omega}$ for $a \in \mathfrak{B}^{* *}$ and $b \in\left(\mathfrak{B}^{* *}\right)_{\omega}^{\prime}$ are also as in that lemma. For $e \in \mathfrak{B} \cap\left(\mathfrak{B}^{* *}\right)_{\omega}^{\prime}$ we will have:

$$
\begin{aligned}
\mid\left\langleP \left[ c^{*} c(I \otimes b)\right.\right. & \left.\left.-\left(I \otimes e^{*}\right) c^{*} c(I \otimes b)\right], \psi\right\rangle \mid \\
& =\left|\left\langle P\left[c^{*} c(v \otimes b)-\left(I \otimes e^{*}\right) c^{*} c(v \otimes b)\right], \varphi\right\rangle\right| \\
& =\left|\left\langle c^{*} c(v \otimes b)-\left(I \otimes e^{*}\right) c^{*} c(v \otimes b), \varphi \otimes \omega\right\rangle\right| \\
& =\left|\left\langle\left(\pi_{\varphi} \otimes \pi\right)(c) v \tilde{\otimes} b, \tilde{c}-\left(\pi_{\varphi} \otimes \pi\right)(c) I \tilde{\otimes} e\right\rangle\right| \\
& =\left|\left\langle\left(\pi_{\varphi} \otimes \pi\right)(c(v \otimes I)) I \tilde{\otimes} b, \tilde{c}-I \otimes \pi^{\prime}(e) \tilde{c}\right\rangle\right| \\
& =\left|\left\langle\left(I \otimes\left(I-\pi^{\prime}(e)^{*}\right) \pi^{\prime}(b)\right)(c(v \otimes I))^{\tilde{c}}, \tilde{c}\right\rangle\right| \\
& \leqslant\left\|\left(I-\pi^{\prime}(e)^{*}\right) \pi^{\prime}(b)\right\|\left\|(c(v \otimes I))^{-}\right\|\|\tilde{c}\| \\
& \leqslant\left\|b-b e^{*}\right\|\left\langle\left(v^{*} \otimes I\right) c^{*} c(v \otimes I), \varphi \otimes \omega\right\rangle^{1 / 2}\left\langle c^{*} c, \varphi \otimes \omega\right\rangle^{1 / 2} \\
& =\left\|b-b e^{*}\right\|\left\langle v^{*} P\left(c^{*} c\right) v, \varphi\right\rangle^{1 / 2}\left\langle P\left(c^{*} c\right), \varphi\right\rangle^{1 / 2} \\
& \leqslant\left\|b-b e^{*}\right\|\left\|P\left(c^{*} c\right)\right\|\|\varphi\| .
\end{aligned}
$$

Since $\|\varphi\|=\|\psi\|$ we therefore have that

$$
\left\|P\left[c^{*} c(I \otimes b)-\left(I \otimes e^{*}\right) c^{*} c(I \otimes b)\right]\right\| \leqslant\left\|b-b e^{*}\right\|\left\|P\left(c^{*} c\right)\right\| .
$$

We have also here used the formula (1.3) established in Lemma 1.4.

If we now take $e=e_{i}^{*} e_{i}$ where $e_{i}$ runs through an approximate identity for $B$ we see that

$$
P\left(\left(I \otimes e_{i}^{*} e_{i}\right) c^{*} c(I \otimes b)\right) \rightarrow P\left(c^{*} c(I \otimes b)\right) .
$$

$c \in \tilde{M}(\mathfrak{A} \otimes \mathfrak{B})$, so $\left(I \otimes e_{i}\right) c^{*} c \in \mathfrak{A} \otimes \mathfrak{B}$ and therefore

$$
P\left(\left(I \otimes e_{i}^{*}\right)\left(I \otimes e_{i}\right) c^{*} c(I \otimes b)\right) \in \mathfrak{A} .
$$

Thus $P\left(c^{*} c(I \otimes b)\right) \in \mathfrak{A}$.

ReMARK. The map $P_{\omega}$ considered here is an example of so-called operator valued weights studied in [31]. 


\section{Chapter 2. The Duality Theorem for $\boldsymbol{W}^{*}$-Crossed Products}

If $G$ is a locally compact group, let $\mathcal{L}(G)$ be the left ring of $G$, i.e. the von Neumann algebra generated by the left regular representation of $G$ over $L^{2}(G)$. For $x \in G$ we shall also denote by $x$ the corresponding element of $\mathcal{L}(G)$, i.e.

$$
x f(y)=f\left(x^{-1} y\right) \quad \text { for } f \in L^{2}(G), x, y \in G .
$$

Suppose $A$ is a von Neumann algebra acting in a Hilbert space $H$, and let $\rho$ be a $\sigma$-continuous automorphic representation of $G$ over $A$, that is: $\rho$ is a homomorphism from $G$ into the group of *-automorphisms of $A$ such that the map $x \rightarrow \rho_{x}(a)$ is $\sigma$-continuous for all $a \in A$. The triple $(A, \rho, G)$ is then called a covariant system.

Two covariant systems $(A, \rho, G)$ and $(B, \sigma, G)$ over $G$ are said to be equivalent if there is a normal *-isomorphism $\varphi$ of $A$ onto $B$ such that

$$
\varphi\left(\rho_{x}(a)\right)=\sigma_{x}(\varphi(a)) \text { for all } x \in G, a \in A .
$$

Suppose now that we have a covariant system $(A, \rho, G)$ and that $A$ is a concrete von Neumann algebra over some Hilbert space $H$. Define a normal, faithful *-representation $\tilde{\rho}$ of $A$ over $X=L^{2}(G, H)=H \otimes L^{2}(G)$ by

$$
\tilde{\rho}(a) f(x)=\rho_{x^{-1}}(a) f(x) \text { for } a \in A, f \in X, x \in G,
$$

and define a continuous unitary representation $\lambda$ of $G$ over $X$ by

$$
\lambda(x) f(y)=f\left(x^{-1} y\right) \text { for } f \in X, x, y \in G .
$$

It is easy to check that $\lambda(x) \tilde{\rho}(a) \lambda\left(x^{-1}\right)=\tilde{\rho}\left(\rho_{x}(a)\right)$ for $x \in G, a \in A$, so if we define

$$
\tau_{x}(a)=\lambda(x) a \lambda\left(x^{-1}\right) \text { for } x \in G, a \in \tilde{\rho}(A)
$$

we will have that $(A, \rho, G)$ and $(\tilde{\rho}(A), \tau, G)$ are equivalent.

The regular $W^{*}$-crossed product of $A$ and $G$, or the regular covariance algebra of $(A, \rho, G)$ is defined as the von Neumann algebra generated by $\tilde{\rho}(A) \cup \lambda(G)$ and it is denoted by $W_{r}^{*}(A, \rho, G)$. It is straightforward to check that the definition of $W_{r}^{*}(A, \rho, G)$ does not depend on the Hilbert space $H$ on which $A$ is represented.

The following lemma says essentially that the algebraic relation between $W_{r}^{*}(A, \rho, G), A$ and $\mathcal{L}(G)$ is determined by the "innerness" of $\rho$ :

LEMMA 2.1. Suppose $\rho$ is inner, that is there is a continuous unitary representation $U$ of $G$ such that $\rho_{x}(a)=U_{x} a U_{x^{-1}}$ and $U_{x} \in A$ for all $x \in G, a \in A$. Let $U$ be the unitary operator over $L^{2}(G, H)$ defined by

$$
U f(x)=U_{x} f(x) \text { for } x \in G, f \in L^{2}(G, H) .
$$

Then $W_{r}^{*}(A, \rho, G)=U^{*}(A \otimes \mathcal{L}(G)) U$. 
Proof. It is straightforward to check that $\tilde{\rho}(a)=U^{*}(a \otimes I) U$ for $a \in A$ and that

$$
U(I \otimes x) U^{*}=U_{x} \otimes x \text { for } x \in G .
$$

Since $U_{x}$ is supposed to be in $A$, the result follows immediately.

So in order to get interesting examples one should consider automorphisms which are not inner.

Define a unitary operator $W$ over $X \otimes L^{2}(G) \cong L^{2}(G \times G, H)$ by

$$
W f(x, y)=f(x, x y) \text { for } f \in L^{2}(G \times G, H), x, y \in G .
$$

Then it is easy to check that

$$
\begin{array}{ll}
W^{*}(\tilde{\rho}(a) \otimes I) W=\tilde{\rho}(a) \otimes I & \text { for } a \in A, \\
W^{*}(\lambda(x) \otimes I) W=\lambda(x) \otimes x & \text { for } x \in G .
\end{array}
$$

So if $\mathfrak{U}=W_{r}^{*}(G, A)$ we can define a normal isomorphism $\delta: \mathfrak{A} \rightarrow \mathfrak{U} \otimes \mathfrak{L}(G)$ by

$$
\delta(a)=W^{*}(a \otimes I) W \quad \text { for } a \in \mathfrak{A} .
$$

If in particular $A=\mathbf{C}, W_{r}^{*}(\mathbf{C}, G)=\mathcal{L}(G)$, and the corresponding isomorphism $\mathcal{L}(G) \rightarrow \mathcal{L}(G) \otimes \mathcal{L}(G)$ we shall denote by $\delta_{G}$. So for $x \in G$ we have $\delta_{G}(x)=x \otimes x$. The duality theorem for locally compact groups then tells us that $G \cong\left\{u \in \mathcal{L}(G) \backslash\{0\} \mid \delta_{G}(u)=u \otimes u\right\}$ where the latter set is given relative $\sigma$-topology from $\mathcal{L}(G)$ (cf. [26, Theorem 16]). We are however not going to use this fact.

For a general $\mathfrak{A}$ and $\delta$ as constructed above we will have

$$
(\delta \otimes i) \delta(a)=\left(i \otimes \delta_{G}\right) \delta(a) \quad \text { for all } a \in \mathfrak{U} .
$$

( $i$ denotes the identity map both on $\mathfrak{A}$ and on $\mathcal{L}(G)$.) (2.6) follows easily from (2.4) and (2.5), since they imply that (2.6) holds for elements $a \in \tilde{\rho}(A) \cup$ $\lambda(G)$, and this set generates $\mathfrak{A}$.

The duality theorem we are going to prove says essentially that the triple $(\mathfrak{A}, \lambda, \delta)$ satisfying (2.5) and (2.6) in fact characterizes all von Neumann algebras which can be obtained as a crossed product over $G$. More precisely we shall prove:

THEOREM 1. If $\mathfrak{U}$ is a von Neumann algebra and $G$ a locally compact group, then $\mathfrak{A}$ is isomorphic to a regular $W^{*}$-crossed product $W_{r}^{*}(A, \rho, G)$ for some covariant system $(A, \rho, G)$ over $G$ if and only if there is a $\sigma$-continuous homomorphism $\lambda: G \rightarrow \mathfrak{A}_{u}$ and a normal isomorphism $\delta$ from $\mathfrak{A}$ into $\mathfrak{A} \otimes \mathcal{L}(G)$ satisfying

$$
\begin{gathered}
(\delta \otimes i) \delta(a)=\left(i \otimes \delta_{G}\right) \delta(a) \quad \text { for } a \in \mathfrak{U}, \\
\delta \circ \lambda(x)=\lambda(x) \otimes x \quad \text { for } x \in G .
\end{gathered}
$$


$A$ and $\rho$ are uniquely determined by $\delta$ and $\lambda$ up to isomorphisms by:

$$
\begin{aligned}
A & =\{a \in \mathfrak{A} \mid \delta(a)=a \otimes I\}, \\
\rho_{x}(a) & =\lambda(x) a \lambda\left(x^{-1}\right) \quad \text { for } a \in A, x \in G .
\end{aligned}
$$

In proving the uniqueness part we shall need the following result. It follows from the general imprimitivity theorem (cf. [15, Chapter 15]) but we shall give the short proof possible for our simpler situation.

Proposition 2.2. If $(A, \rho, G)$ is a covariant system and $\tilde{\rho}$ and $\lambda$ are as in (2.1) and (2.2) we can also define a normal representation $\tilde{\rho}$ of the abelian von Neumann algebra $L^{\infty}(G)$ over $L^{2}(G, H)$ by

$$
\tilde{\rho}(\varphi) f(x)=\varphi(x) f(x) \quad \text { for } \varphi \in L^{\infty}(G), f \in L^{2}(G, H), x \in G .
$$

Then

$$
\tilde{\rho}(A)^{\prime} \cap \lambda(G)^{\prime} \cap \tilde{\rho}\left(L^{\infty}(G)\right)^{\prime}=A^{\prime} \otimes I .
$$

Proof. It is well known that $\lambda(G)^{\prime} \cap \tilde{\rho}\left(L^{\infty}(G)\right)^{\prime}=\mathscr{B}(H) \otimes I$, and it is easy to check that

$$
A^{\prime} \otimes I \subseteq \tilde{\rho}(A)^{\prime} \cap \lambda(G)^{\prime} \cap \tilde{\rho}\left(L^{\infty}(G)\right)^{\prime}=\tilde{\rho}(A)^{\prime} \cap(\Re(H) \otimes I) .
$$

Now suppose $B \in \tilde{\rho}(A)^{\prime} \cap(\Re(H) \otimes I)$, so $B=b \otimes I$ for some $b \in \Re(H)$. Then for $a \in A, f \in L^{2}(G, H)$

$$
(b \otimes I) \tilde{\rho}(a) f(s)=\tilde{\rho}(a)(b \otimes I) f(s)
$$

i.e.

$$
b \rho_{s^{-1}}(a) f(s)=\rho_{s^{-1}}(a) b f(s),
$$

equality meaning equality as elements of $L^{2}(G, H)$. Since this shall hold for all $f \in L^{2}(G, H)$ we must have $b a=a b$ for all $a \in A$, i.e. $b \in A^{\prime}$. Thus $A^{\prime} \otimes I=\tilde{\rho}(A)^{\prime} \cap \lambda(G)^{\prime} \cap \tilde{\rho}\left(L^{\infty}(G)\right)^{\prime}$.

Returning to the proof of the uniqueness part of Theorem 1 let us suppose that $(A, \rho, G)$ is given and that $\mathfrak{A}=W_{r}^{*}(A, \rho, G)$ and $\lambda, \delta$ are constructed as above. Our goal is to prove that

$$
\tilde{\rho}(A)=\{a \in \mathfrak{A} \mid \delta(a)=a \otimes I\} .
$$

Without loss of generality we can assume that $\rho_{x}$ is unitarily implemented over $H$, because if this was not the case we could replace $(A, \rho, G)$ by the equivalent system $(\tilde{\rho}(A), \tau, G)$ defined in the beginning of this chapter. So we shall assume that we have a continuous unitary representation $U$ of $G$ over $H$ such that

$$
\rho_{x}(a)=U_{x} a U_{x^{-1}} \text { for } a \in A, x \in G .
$$

Let $\tilde{G}$ be the reverse group of $G$, i.e. $G=\tilde{G}$ as topological spaces, but multiplication in $\tilde{G}$ is defined by $x \cdot y=y x$. (We shall write multiplication in 
$\tilde{G}$ by a dot, multiplication in $G$ by juxtaposition.) We can now define a $\sigma$-continuous automorphic representation $\rho^{\prime}$ of $\tilde{G}$ on $A^{\prime}$ by

$$
\rho_{x}^{\prime}\left(a^{\prime}\right)=U_{x^{-1}} a^{\prime} U_{x} \quad \text { for } a^{\prime} \in A^{\prime}, x \in \tilde{G} .
$$

Just as before we will get representations $\tilde{\rho}^{\prime}, \lambda^{\prime}$ and $\tilde{\rho}^{\prime}$ of $A^{\prime}, \tilde{G}$ and $L^{\infty}(\tilde{G})$ respectively over $L^{2}(\tilde{G}, H)$ by

$$
\begin{aligned}
& \tilde{\rho}^{\prime}\left(a^{\prime}\right) f(x)=\rho_{x^{-1}}^{\prime}\left(a^{\prime}\right) f(x)=U_{x} a^{\prime} U_{x^{-1}} f(x), \\
& \lambda^{\prime}(y) f(x)=f\left(y^{-1} \cdot x\right)=f\left(x y^{-1}\right), \\
& \rho^{\prime}(\varphi) f(x)=\varphi(x) f(x),
\end{aligned}
$$

for $a^{\prime} \in A^{\prime}, \varphi \in L^{\infty}(\tilde{G}), f \in L^{2}(\tilde{G}, H), x, y \in \tilde{G}$.

From Proposition 2.2 it now follows that

$$
A \otimes I=\tilde{\rho}^{\prime}\left(A^{\prime}\right)^{\prime} \cap \lambda^{\prime}(\tilde{G})^{\prime} \cap \tilde{\rho}^{\prime}\left(L^{\infty}(\tilde{G})\right)^{\prime} .
$$

Define $U: L^{2}(\tilde{G}, H) \rightarrow L^{2}(G, H)$ by

$$
U f(x)=\Delta(x)^{-1 / 2} U_{x^{-1}} f(x) \text { for } f \in L^{2}(\tilde{G}, H), x \in G .
$$

Noting that the left Haar measure $d \tilde{x}$ in $\tilde{G}$ is nothing but the right Haar measure $\Delta\left(x^{-1}\right) d x$ in $G$, we have

$$
\|U f\|^{2}=\int_{G} \Delta\left(x^{-1}\right)\left\|U_{x^{-1}} f(x)\right\|^{2} d x=\int_{\tilde{G}}\|f(x)\|^{2} d \tilde{x}=\|f\|^{2} .
$$

So $U$ is unitary. It is now straightforward to check the following formulas:

$$
\begin{gathered}
U(a \otimes I) U^{*}=\tilde{\rho}(a) \text { for } a \in A \\
U \tilde{\rho}^{\prime}\left(a^{\prime}\right) U^{*}=a^{\prime} \otimes I \text { for } a^{\prime} \in A^{\prime}, \\
U \lambda^{\prime}(y) U^{*} f(x)=\Delta(y)^{-1 / 2} U_{y^{-1}} f\left(x y^{-1}\right) \quad \text { for } f \in L^{2}(G, H), x, y \in G, \\
U \tilde{\rho}^{\prime}(\varphi) U^{*}=\tilde{\rho}(\varphi) \text { for } \varphi \in L^{\infty}(\tilde{G})=L^{\infty}(G) .
\end{gathered}
$$

If we define $\pi(y)=U \lambda^{\prime}\left(y^{-1}\right) U^{*}$ for $y \in G, \pi$ is a continuous representation of $G$ over $L^{2}(G, H)$, and from (2.8) and (2.9)-(2.12) we have that

$$
\tilde{\rho}(A)=\left(A^{\prime} \otimes I\right)^{\prime} \cap \pi(G)^{\prime} \cap \tilde{\rho}\left(L^{\infty}(G)\right)^{\prime} .
$$

Proposition 2.3. With notation as above

$$
\tilde{\rho}(A)=\{a \in \mathfrak{U} \mid \delta(a)=a \otimes I\} .
$$

Proof. We have already seen in (2.4) that $\tilde{\rho}(A) \subset\{a \in \mathfrak{A} \mid \delta(a)=a \otimes I\}$, by using (2.13) we shall prove the reverse inclusion.

$$
\tilde{\rho}(A) \subseteq\left(A^{\prime} \otimes I\right)^{\prime} \text { so } \delta(\tilde{\rho}(A))=\tilde{\rho}(A) \otimes I \subseteq\left(A^{\prime} \otimes I \otimes I\right)^{\prime} .
$$

It is straightforward to check that also

$$
\delta \circ \lambda(x)=\lambda(x) \otimes x \in\left(A^{\prime} \otimes I \otimes I\right)^{\prime} \quad \text { for all } x \in G,
$$


hence $\delta \circ \lambda(G) \subset\left(A^{\prime} \otimes I \otimes I\right)^{\prime}$. Thus also $\delta(\mathfrak{U}) \subseteq\left(A^{\prime} \otimes I \otimes I\right)^{\prime}$, so if $a$ is an element from $\mathfrak{A}$ with $\delta(a)=a \otimes I$ we must have that

$$
a \otimes I \in\left(A^{\prime} \otimes I \otimes I\right)^{\prime}
$$

i.e.

$$
a \in\left(A^{\prime} \otimes I\right)^{\prime} \quad \text { for all } a \in \mathfrak{A} \text { with } \delta(a)=a \otimes I .
$$

Next, for $a \in A, x, y \in G$ we have $\tilde{\rho}(a) \pi(y)=\pi(y) \tilde{\rho}(a)$ and $\lambda(x) \pi(y)=$ $\pi(y) \lambda(x)$. So $\tilde{\rho}(A) \cup \lambda(G) \subseteq \pi(G)^{\prime}$, hence

$$
\mathfrak{A} \subseteq \pi(G)^{\prime} .
$$

We shall identify an element $\varphi \in L^{\infty}(G)$ with the operator $\varphi$ over $L^{2}(G)$ defined by $\varphi f(x)=\varphi(x) f(x)$ for $f \in L^{2}(G)$. Then obviously $I \otimes I \otimes \varphi \in$ $(\mathfrak{A} \otimes I)^{\prime}$, so $W^{*}(I \otimes I \otimes \varphi) W \in \delta(\mathfrak{U})^{\prime}$. Taking $\tilde{\tilde{\rho}}(\varphi)=W^{*}(I \otimes I \otimes \varphi) W$ we see that $\tilde{\tilde{\rho}}(\varphi) f(s, t)=\varphi\left(s^{-1} t\right) f(s, t)$. So if we take an element $a \in \mathfrak{A}$ with $\delta(a)=a \otimes I, \quad$ we have $(a \otimes I) \tilde{\tilde{\rho}}(\varphi)=\tilde{\tilde{\rho}}(\varphi)(a \otimes I)$, i.e. for all $\xi, \eta \in$ $L^{2}(G, H), f, g \in L^{2}(G)$ we have

$$
\langle(a \otimes I) \tilde{\tilde{\rho}}(\varphi)(\xi \otimes f), \eta \otimes g\rangle=\langle\tilde{\tilde{\rho}}(\varphi)(a \otimes I)(\xi \otimes f), \eta \otimes g\rangle .
$$

Thus

$$
\begin{aligned}
\int_{G} \int_{G} \varphi\left(s^{-1} t\right)\left\langle\xi(s), a^{*} \eta(s)\right\rangle f(t) \overline{g(t)} d s d t \\
=\int_{G} \int_{G} \varphi\left(s^{-1} t\right)\langle a \xi(s), \eta(s)\rangle f(t) \overline{g(t)} d s d t .
\end{aligned}
$$

Since this shall hold for all $f, g \in L^{2}(G)$, we have

$$
\int_{G} \varphi\left(s^{-1} t\right)\left\langle\xi(s), a^{*} \eta(s)\right\rangle d s=\int_{G} \varphi\left(s^{-1} t\right)\langle a \xi(s), \eta(s)\rangle d s
$$

for all $\varphi \in L^{\infty}(G)$ and $t \in G$. In particular, if we take $t=e$ and replace $\varphi$ by the function $s \rightarrow \varphi\left(s^{-1}\right)$ we get that

$$
\int_{G} \varphi(s)\left\langle\xi(s), a^{*} \eta(s)\right\rangle d s=\int_{G} \varphi(s)\langle a \xi(s), \eta(s)\rangle d s
$$

so $\left\langle\tilde{\rho}(\varphi) \xi, a^{*} \eta\right\rangle=\langle\tilde{\rho}(\varphi) a \xi, \eta\rangle$. This is for all $\xi, \eta \in L^{2}(G, H)$, so $a \tilde{\rho}(\varphi)=$ $\tilde{\rho}(\varphi) a$ for all $\varphi \in L^{\infty}(G)$. So we have proved:

$$
a \in \tilde{\rho}\left(L^{\infty}(G)\right)^{\prime} \quad \text { if } a \in \mathfrak{A} \text { with } \delta(a)=a \otimes I .
$$

(2.13) together with (2.14), (2.15) and (2.16) give us that $\{a \in \mathfrak{U} \mid \delta(a)=a \otimes$ $I\} \subseteq \tilde{\rho}(A)$, and the proposition is proved. I am indepted to J.M.G. Fell for the main idea of this proof, to use Proposition 2.2 on the covariant system $\left(A^{\prime}, \rho^{\prime}, \tilde{G}\right)$.

Proof of uniqueness in Theorem 1. This will now follow easily, because if $\mathfrak{U}=W_{r}^{*}(A, \rho, G)$ we just proved that $A \cong \tilde{\rho}(A)=\{a \in \mathfrak{A} \mid \delta(a)=a \otimes I\}$ and if we define $\tau: G \rightarrow \operatorname{Aut}(\tilde{\rho}(A))$ by $\tau_{x}(\tilde{\rho}(a))=\lambda(x) \tilde{\rho}(a) \lambda\left(x^{-1}\right)$ for $x \in G$, 
$a \in A$, we have $\tau_{x}(\tilde{\rho}(a))=\tilde{\rho}\left(\rho_{x}(a)\right)$ for $x \in G, a \in A$, so the covariant systems $(A, \rho, G)$ and $(\tilde{\rho}(A), \tau, G)$ are equivalent.

Let us now turn to the other part of Theorem 1, so we shall suppose we have a von Neumann algebra $\mathfrak{A}$, a normal isomorphism $\delta: \mathfrak{A} \rightarrow \mathfrak{U} \otimes \mathfrak{L}(G)$ and a continuous homomorphism $\lambda: G \rightarrow \mathfrak{A}_{u}$ satisfying

$$
\begin{aligned}
(\delta \otimes i) \delta(a) & =\left(i \otimes \delta_{G}\right) \delta(a) \quad \text { for all } a \in \mathfrak{A}, \\
\delta \circ \lambda(x) & =\lambda(x) \otimes x \quad \text { for } x \in G .
\end{aligned}
$$

A pair $(\mathfrak{A}, \delta)$ satisfying $(2.6)$ is called a $\mathscr{L}(G)$-comodule. Let $\mathfrak{A}_{*}$ be the predual of $\mathfrak{A}, A(G)=\mathfrak{L}(G)_{*}$ the predual of $\mathfrak{L}(G) . A(G)$ is usually called the Fourier-algebra of $G$, and is a commutative Banach *-algebra as follows: If $\alpha, \beta \in A(G)$ we define $\alpha \beta$ by

$$
\langle a, \alpha \beta\rangle=\left\langle\delta_{G}(a), \alpha \otimes \beta\right\rangle \text { for all } a \in \mathcal{L}(G)
$$

and $\alpha^{*}$ is defined by

$$
\left\langle a, \alpha^{*}\right\rangle=\overline{\langle C a C, \alpha\rangle} \text { for all } a \in \mathfrak{L}(G),
$$

where $C: L^{2}(G) \rightarrow L^{2}(G)$ is the conjugate linear map defined by $C f(x)=\overline{f(x)}$.

$A(G)$ can be concretely realized as follows: If $\alpha \in A(G)$ there are functions $\xi, \eta \in L^{2}(G)$ such that $\langle a, \alpha\rangle=\langle a \xi, \eta\rangle$ for all $a \in \mathcal{L}(G)$. Define a function in $C_{0}(G)$ by

$$
\alpha(x)=\langle x \xi, \eta\rangle \text { for } x \in G,
$$

we then get an embedding (not an isometric one) of $A(G)$ into $C_{0}(G)$ and it turns out that the multiplication and involution just defined in $A(G)$ under this embedding corresponds to pointwise multiplication and complex conjugation of functions in $C_{0}(G)$. We can also define an action of $A(G)$ on $\mathfrak{A}_{*}$ by

$$
\langle a, \varphi \alpha\rangle=\langle\delta(a), \varphi \otimes \alpha\rangle \text { for } a \in \mathfrak{A}, \varphi \in \mathfrak{A}_{*}, \alpha \in A(G) .
$$

(2.6) will imply that this really is an action, i.e. that $(\varphi \alpha) \beta=\varphi(\alpha \beta)$ for $\varphi \in \mathfrak{A}_{*}, \alpha, \beta \in A(G)$. We also see that

$$
\|\varphi \alpha\| \leqslant\|\varphi\|\|\alpha\| \text { for } \varphi \in \mathfrak{A}_{*}, \alpha \in A(G) .
$$

Lemma 2.4. The linear span of $\left\{\varphi \alpha \mid \varphi \in \mathfrak{A}_{*}, \alpha \in A(G)\right\}$ is norm dense in $\mathfrak{A}_{*}$.

Proof. If this was not the case, there would be a nonzero $a \in \mathfrak{A}$ with

$$
\langle a, \varphi \boldsymbol{\alpha}\rangle=0 \text { for all } \varphi \in \mathfrak{A}_{*}, \alpha \in A(G)
$$

i.e. $\langle\delta(a), \varphi \otimes \alpha\rangle=0$. Since $\left\{\varphi \otimes \alpha \mid \varphi \in \mathfrak{A}_{*}, \alpha \in A(G)\right\}$ spans a normdense subspace of $(\mathfrak{A} \otimes \mathfrak{L}(G))_{*}$, we must have $\delta(a)=0$. This is a contradiction since $\delta$ is an isomorphism.

If $G$ is an amenable group it is known that $A(G)$ has an approximate 
identity $\{\beta\}$ of the following type: For every $\alpha \in A(G)$ and $\varepsilon>0$ there is a $\beta \in A(G)$ with $\|\beta\|=1$ and $\|\alpha-\alpha \beta\|<\varepsilon$ (see [7]). If we combine Lemma 2.4 with this observation, we get:

LEMMA 2.5. If $G$ is an amenable group, $\varphi \in \mathfrak{A}_{*}$ and $\varepsilon>0$, there exists $\beta \in A(G)$ with $\|\beta\|=1$ and $\|\varphi-\varphi \beta\|<\varepsilon$.

Proof. If $\varphi \in \mathfrak{A}_{*}$ and $\varepsilon>0$, we know from Lemma 2.4 that there are $\varphi_{1}, \ldots, \varphi_{n} \in \mathfrak{A}_{*}$ and $\alpha_{1}, \ldots, \alpha_{n} \in A(G)$ such that

$$
\left\|\sum_{i=1}^{n} \varphi_{i} \alpha_{i}-\varphi\right\|<\frac{\varepsilon}{3},
$$

and there is a $\beta \in A(G)$ with $\|\beta\|=1$ and

$$
\left\|\alpha_{i}-\alpha_{i} \beta\right\|<\varepsilon\left(3 \sum_{i=1}^{n}\left\|\varphi_{i}\right\|\right)^{-1} \text { for } i=1, \ldots, n,
$$

so

$$
\begin{aligned}
\|\varphi-\varphi \beta\| \leqslant & \left\|\varphi-\sum_{i=1}^{n} \varphi_{i} \alpha_{i}\right\|+\left\|\sum_{i=1}^{n} \varphi_{i}\left(\alpha_{i}-\alpha_{i} \beta\right)\right\| \\
& +\left\|\left(\sum_{i=1}^{n} \varphi_{i} \alpha_{i}-\varphi\right) \beta\right\|<\varepsilon / 3+\varepsilon / 3+\varepsilon / 3\|\beta\|=\varepsilon .
\end{aligned}
$$

Definition of the Plancherel weight on $\mathcal{L}(G) . C_{00}(G)$ is a left generalized Hilbert algebra if we give it the inner product from $L^{2}(G)$ and defines multiplication and involution as usual by

$$
\begin{gathered}
f g(x)=\int_{G} f(y) g\left(y^{-1} x\right) d y \quad \text { for } f, g \in C_{00}(G), \\
f^{*}(x)=\Delta\left(x^{-1}\right) \overline{f\left(x^{-1}\right)} \quad \text { for } f \in C_{00}(G) .
\end{gathered}
$$

If we proceed as described in Chapter 1 we get a ${ }^{*}$-representation of $C_{00}(G)$ over $L^{2}(G)$ which generates the von Neumann algebra $\mathcal{L}(G)$. From Chapter 1 it follows that we have a faithful, normal, semifinite weight $\operatorname{tr}$ on $\mathcal{L}(G)^{+}$such that

$$
\operatorname{tr}\left(g^{*} f\right)=\langle f, g\rangle=g^{*} f(e) \quad \text { for } f, g \in C_{00}(G) .
$$

(We identify an element $f \in C_{00}(G)$ with its image in $\mathcal{L}(G)$, which acts on $L^{2}(G)$ by the formula (2.17) for all $g \in L^{2}(G)$.) tr is a trace on $\mathcal{L}(G)$ if and only if $G$ is unimodular, we shall use the notation tr even though it is not a trace in general, since we only shall need its properties as a weight.

We shall now apply the theory developed in Chapter 1 with $\mathfrak{B}=\mathscr{L}(G)$ and 
$\omega=\operatorname{tr}$. Then

$$
\begin{aligned}
& (\mathfrak{U} \otimes \mathscr{L}(G))_{\mathrm{tr}}^{+}=\left\{x \in(\mathfrak{U} \otimes \mathscr{L}(G))^{+} \mid \exists K_{x} \geqslant 0,\right. \\
& \left.\varphi \otimes \operatorname{tr}(x) \leqslant K_{x}\|\varphi\| \quad \text { for all } \varphi \in \mathfrak{A}_{*}^{+}\right\}, \\
& \widetilde{\mathfrak{S}}_{\mathrm{tr}}=\left\{x \in \mathfrak{A} \otimes \mathfrak{L}(G) \mid x^{*} x \in(\mathfrak{A} \otimes \mathscr{L}(G))_{\mathrm{tr}}^{+}\right\},
\end{aligned}
$$

and

$$
(\mathfrak{A} \otimes \mathscr{L}(G))_{\mathrm{tr}}=\operatorname{linspan}(\mathfrak{U} \otimes \mathfrak{L}(G))_{\mathrm{tr}}^{+}=\mathfrak{\mathfrak { V }}_{\mathrm{tr}}^{*} \mathfrak{V}_{\mathrm{tr}} .
$$

The corresponding map $P_{\mathrm{tr}}:(\mathfrak{A} \otimes \mathscr{L}(G))_{\mathrm{tr}} \rightarrow \mathfrak{A}$ defined by $\left\langle P_{\mathrm{tr}}(x), \varphi\right\rangle=$ $\langle x, \varphi \otimes \operatorname{tr}\rangle$ for all $\varphi \in \mathfrak{A}_{*}$, we shall simply denote by $P$. In the same way we also get a map $q:(\mathfrak{H} \otimes \mathfrak{L}(G) \otimes \mathfrak{L}(G))_{\mathrm{tr}} \rightarrow \mathfrak{A} \otimes \mathfrak{L}(G)$ defined by $\langle q(x), \varphi\rangle=$ $\langle x, \varphi \otimes \operatorname{tr}\rangle$ for $x \in(\mathfrak{A} \otimes \mathfrak{L}(G) \otimes \mathfrak{L}(G))_{\mathrm{tr}}, \varphi \in(\mathfrak{A} \otimes \mathfrak{L}(G))_{*}$. Define $\mathfrak{A}_{0}=$ $\delta^{-1}\left(\mathfrak{I}_{\mathrm{tr}}\right), \mathfrak{A}_{1}=\delta^{-1}\left((\mathfrak{A} \otimes \mathfrak{L}(G))_{\mathrm{tr}}\right)$, then $\mathfrak{A} \mathfrak{A}_{0} \subseteq \mathfrak{A}_{0}$ and $\mathfrak{A}_{1}=\mathfrak{U}_{0}^{*} \mathfrak{A}_{0}$.

Lemma 2.6. $\mathfrak{A}_{1}$ is $\sigma$-dense in $\mathfrak{A}$.

Proof. If $a \in \mathfrak{A}$, there is a net $f_{i}$ from $C_{00}(G)$ such that $\lambda\left(f_{i}^{*}\right) a \lambda\left(f_{i}\right) \rightarrow a$ in the $\sigma$-topology, so it is sufficient to prove that $\lambda(f) \in \mathfrak{A}_{0}$ for $f \in C_{00}(G)$. For $\varphi \in \mathfrak{A}_{*}^{+}$,

$$
\begin{aligned}
\left\langle\delta\left(\lambda(f)^{*} \lambda(f)\right), \varphi \otimes \operatorname{tr}\right\rangle & =\left\langle\int f^{*} f(x) \lambda(x) \otimes x d x, \varphi \otimes \operatorname{tr}\right\rangle \\
& =f^{*} f(e) \varphi(I)=\langle f, f\rangle\|\varphi\| .
\end{aligned}
$$

So $\delta \circ \lambda(f) \in \widetilde{\mathfrak{V}}_{\text {tr }}$, hence $\lambda(f) \in \mathfrak{A}_{0}$. Note that we now also have proved that $P\left(\delta\left(\lambda\left(f^{*} f\right)\right)\right)=f^{*} f(e) I$ for $f \in C_{00}(G)$.

Lemma 2.7. For $x \in(\mathfrak{A} \otimes \mathcal{L}(G))_{\mathrm{tr}}$ we have

$$
\begin{gathered}
\delta \circ P(x)=q(\delta \otimes i)(x), \\
q\left(i \otimes \delta_{G}\right)(x)=P(x) \otimes I .
\end{gathered}
$$

Proof. Suppose first that $x=a \otimes f$ with $a \in \mathfrak{A}, f \in C_{00}(G)^{*} C_{00}(G)$. Then

$$
\begin{aligned}
\delta \circ P(x)=\delta & \circ P(a \otimes f)=\delta(f(e) a)=f(e) \delta(a) \\
= & q(\delta(a) \otimes f)=q(\delta \otimes i)(a \otimes f)=q(\delta \otimes i)(x), \\
q\left(i \otimes \delta_{G}\right)(x) & =q\left(i \otimes \delta_{G}\right)(a \otimes f)=q\left(a \otimes \delta_{G}(f)\right) \\
= & q\left(a \otimes \int_{G} f(x) x \otimes x d x\right)=a \otimes f(e) I \\
& =f(e) a \otimes I=P(a \otimes f) \otimes I=P(x) \otimes I
\end{aligned}
$$

so at least for elements $x$ of this form (2.19) and (2.20) hold. 
Suppose $b \in \mathfrak{A} \otimes_{\text {alg }} C_{00}(G)$ (the algebraic tensor product) and that $x \in \mathfrak{A}$ $\otimes \mathscr{L}(G)$. By the Kaplansky density theorem there is a net from $\mathfrak{A} \otimes C_{00}(G)$ with $\left\|x_{i}\right\| \leqslant\|x\|$ such that $x_{i} \rightarrow x$ in the $\sigma$-topology. Since (2.19) and (2.20) hold for $b^{*} x_{i} b$ it follows from Corollary 1.3 that the formulas also hold for $b^{*} x b$.

Before proving that (2.19) and (2.20) hold for all $x \in(\mathscr{A} \otimes \mathscr{L}(G))_{\mathrm{tr}}$ let us first check that we will have $\delta \otimes i(x)$ and $i \otimes \delta_{G}(x)$ in $(\mathfrak{A} \otimes \mathscr{L}(G) \otimes E(G))_{\mathrm{tr}}$. It suffices to prove this for $x \geqslant 0$.

$$
\begin{aligned}
\left|\left\langle\delta \otimes i(x), \sum_{i=1}^{n} \varphi_{i} \otimes \alpha_{i} \otimes \operatorname{tr}\right\rangle\right| & =\left|\left\langle x, \sum_{i=1}^{n} \varphi_{i} \alpha_{i} \otimes \operatorname{tr}\right\rangle\right| \\
& \leqslant K_{x}\left\|\sum_{i=1}^{n} \varphi_{i} \alpha_{i}\right\| \leqslant K_{x}\left\|\sum_{i=1}^{n} \varphi_{i} \otimes \alpha_{i}\right\|
\end{aligned}
$$

for all $\varphi_{1}, \ldots, \varphi_{n} \in \mathfrak{A}_{*}, \alpha_{1}, \ldots, \alpha_{n} \in A(G)$, so

$|\langle\delta \otimes i(x), \varphi \otimes \operatorname{tr}\rangle| \leqslant K_{x}\|\varphi\| \quad$ for all $\varphi \in(\mathfrak{A} \otimes \mathcal{L}(G))_{*}$,

so $\delta \otimes i(x) \in(\mathfrak{A} \otimes \mathfrak{L}(G) \otimes \mathfrak{L}(G))_{\mathrm{tr}}^{+}$.

$$
\begin{aligned}
\mid\langle i \otimes & \left.\delta_{G}(x), \sum_{i=1}^{n} \varphi_{i} \otimes \alpha_{i} \otimes \operatorname{tr}\right\rangle|=|\left\langle x, \sum_{i=1}^{n} \varphi_{i} \otimes\left(\alpha_{i} \otimes \operatorname{tr}\right) \circ \delta_{G}\right\rangle \mid \\
& =\left|\left\langle x, \sum_{i=1}^{n} \alpha_{i}(e) \varphi_{i} \otimes \operatorname{tr}\right\rangle\right| \leqslant K_{x}\left\|\sum_{i=1}^{n} \alpha_{i}(e) \varphi_{i}\right\| \leqslant K_{x}\left\|\sum_{i=1}^{n} \varphi_{i} \otimes \alpha_{i}\right\| .
\end{aligned}
$$

Here we have used the fact that $(\alpha \otimes \operatorname{tr}) \circ \delta_{G}=\alpha(e) \operatorname{tr}$ for $\alpha \in A(G)$. Thus

$$
\left\langle i \otimes \delta_{G}(x), \varphi \otimes \operatorname{tr}\right\rangle \mid \leqslant K_{x}\|\varphi\| \quad \text { for all } \varphi \in(\mathscr{U} \otimes \mathscr{L}(G))_{*}
$$

so $i \otimes \delta_{G}(x) \in(\mathfrak{A} \otimes \mathfrak{L}(G) \otimes \mathfrak{L}(G))_{\mathrm{tr}}^{+}$.

We now want to prove that (2.19) and (2.20) hold for all $x \in(\mathscr{H} \otimes E(G))_{\mathrm{tr}}$, and it obviously suffices to prove this for $x \geqslant 0$. Take a bounded net $\left\{f_{i}\right\}$ from $C_{00}(G)$ such that $f_{i} \rightarrow I$ in the $\sigma$-topology. Then (2.19) and (2.20) hold for $\left(I \otimes f_{i}^{*}\right) x\left(I \otimes f_{i}\right)$, and from Lemma 1.4 it follows that (2.19) and (2.20) also hold for $x$.

Now let $A=\{a \in \mathfrak{A} \mid \delta(a)=a \otimes I\}$, obviously $\lambda(x) A \lambda\left(x^{-1}\right)=A$ for all $x \in G$, so we can define a $\sigma$-continuous automorphic representation $\rho$ of $G$ on $A$ by $\rho_{x}(a)=\lambda(x) a \lambda\left(x^{-1}\right)$ for $a \in A, x \in G$.

Lemma 2.8. $P \circ \delta\left(\mathfrak{A}_{1}\right)=A, A \mathfrak{A}_{1} A \subseteq \mathfrak{A}_{1}$ and $P \circ \delta(a b c)=a P \circ \delta(b) c$ for $a$, $c \in A, b \in \mathfrak{U}_{1}$.

Proof. If $a \in \mathfrak{A}_{1}$ then $\delta(a) \in(\mathfrak{A} \otimes \mathscr{L}(G))_{\mathrm{tr}}$ and from (2.19) and (2.20) we see that

$$
\delta \circ P \circ \delta(a)=q \circ(\delta \otimes i) \circ \delta(a)=q \circ\left(i \otimes \delta_{G}\right) \circ \delta(a)=P \circ \delta(a) \otimes I
$$


so $P \circ \delta(a) \in A$, and thus $P \circ \delta\left(\mathfrak{A}_{1}\right) \subseteq A . A \mathfrak{A}_{1} A=A \mathfrak{A}_{0}^{*} \mathfrak{U}_{0} A$, so to prove that this set is contained in $\mathfrak{U}_{1}$ it suffices to prove that $a b \in \mathfrak{U}_{0}$ for $a \in \mathfrak{U}_{0}$, $b \in A$. For $\varphi \in \mathfrak{A}_{*}^{+}$,

$$
\begin{aligned}
\left\langle\delta\left(b^{*} a^{*} a b\right), \varphi \otimes \operatorname{tr}\right\rangle & =\left\langle\left(b^{*} \otimes I\right) \delta\left(a^{*} a\right)(b \otimes I), \varphi \otimes \operatorname{tr}\right\rangle \\
& =\left\langle\delta\left(a^{*} a\right), \psi \otimes \operatorname{tr}\right\rangle \leqslant K_{a}\|\psi\|
\end{aligned}
$$

where $\psi \in \mathfrak{A}_{*}^{+}$is defined by $\psi(x)=\varphi\left(b^{*} x b\right)$ for $x \in \mathfrak{A}$. So $\|\psi\|=\varphi\left(b^{*} b\right)$ $\leqslant\|b\|^{2}\|\varphi\|$, thus $\left\langle\delta\left(b^{*} a^{*} a b\right), \varphi \otimes \operatorname{tr}\right\rangle \leqslant K_{a}\|b\|^{2}\|\varphi\|$ for all $\varphi \in \mathfrak{A}_{*}^{+}$. Thus $\delta\left(b^{*} a^{*} a b\right) \in(\mathfrak{A} \otimes \mathcal{L}(G))_{\mathrm{tr}}^{+}$, so $b^{*} a^{*} a b \in \mathfrak{A}_{1}^{+}$.

$$
\begin{aligned}
\left\langle P \circ \delta\left(b^{*} a^{*} a b\right), \varphi\right\rangle & =\left\langle\delta\left(b^{*} a^{*} a b\right), \varphi \otimes \operatorname{tr}\right\rangle=\left\langle\delta\left(a^{*} a\right), \psi \otimes \operatorname{tr}\right\rangle \\
& =\left\langle P \circ \delta\left(a^{*} a\right), \psi\right\rangle=\left\langle b^{*} P \circ \delta\left(a^{*} a\right) b, \varphi\right\rangle
\end{aligned}
$$

so $P \circ \delta\left(b^{*} a^{*} a b\right)=b^{*} P \circ \delta\left(a^{*} a\right) b$, and therefore we will also have that $P \circ \delta(a b c)=a P \circ \delta(b) c$ for all $a, c \in A, b \in \mathfrak{A}_{1}$.

It will now follow that $P \circ \delta\left(\mathfrak{A}_{1}\right)=A$, because if $a \in A$, take $f \in C_{00}(G)$ with $\operatorname{tr}\left(f^{*} f\right)=f^{*} f(e)=1$. Then $a \lambda\left(f^{*} f\right) \in \mathfrak{U}_{1}$ and $P \circ \delta\left(a \lambda\left(f^{*} f\right)\right)=$ $a P \circ \delta\left(\lambda\left(f^{*} f\right)\right)=a$.

So to summarize, we see that the linear map $P \circ \delta$ maps the $\sigma$-dense subset $\mathfrak{A}_{1}$ of $\mathfrak{U}$ onto $A$ such that

(i) $P \circ \delta$ is selfadjoint, i.e. $P \circ \delta\left(a^{*}\right)=P \circ \delta(a)^{*}$,

(ii) $P \circ \delta$ is positive, i.e. $P \circ \delta\left(a^{*} a\right) \geqslant 0$,

(iii) $P \circ \delta(a b c)=a P \circ \delta(b) c$ for $a, c \in A, b \in \mathfrak{A}_{1}$,

(iv) For $c \in \mathfrak{A}_{0}$, the map $a \rightarrow P \circ \delta\left(c^{*} a c\right)$ is $\sigma$-continuous on bounded spheres.

So $P \circ \delta$ can be considered as a generalized conditional expectation between $\mathfrak{A}$ and $A$ (cf. [18]).

Lemma 2.9. For $a \in \mathfrak{A}, \varphi, \psi \in C_{00}(G), \xi \in H, x \in G$,

$$
\begin{gathered}
P \circ \delta\left(\lambda(\psi)^{*} a \lambda(\varphi) \lambda\left(x^{-1}\right)\right) \lambda(x) \xi=\Delta(x) \delta\left(\lambda(\psi)^{*} a\right) U(\xi \otimes \varphi)(x), \\
\lambda\left(x^{-1}\right) P \circ \delta\left(\lambda(x) \lambda(\psi)^{*} a \lambda(\varphi)\right) \xi=\delta\left(\lambda(\psi)^{*} a\right) U(\xi \otimes \varphi)\left(x^{-1}\right),
\end{gathered}
$$

where $U$ is the unitary operator over $L^{2}(G, H)$ defined by $U f(x)=\lambda(x) f(x)$ for $f \in L^{2}(G, H)$.

Proof. If $a \in \mathfrak{A}, f \in C_{00}(G)$ we have

$$
\begin{aligned}
P\left[\delta \circ \lambda\left(\psi^{*}\right)(a \otimes f) \delta\left(\lambda(\varphi) \lambda\left(x^{-1}\right)\right)\right] \lambda(x) \xi \\
=P\left[\delta \circ \lambda\left(\psi^{*}\right)(a \otimes f) \delta \circ \lambda(\varphi)\left(I \otimes x^{-1}\right)\right] \xi \\
=P\left[\iiint \psi^{*}(y) f(z) \varphi(u)(\lambda(y) \otimes y)(a \otimes z)\right. \\
\left.\cdot(\lambda(u) \otimes u)\left(I \otimes x^{-1}\right) d u d z d y\right] \xi \quad\left(u \rightarrow z^{-1} y^{-1} u x\right)
\end{aligned}
$$


$=P\left[\Delta(x) \iiint \psi^{*}(y) f(z) \varphi\left(z^{-1} y^{-1} u x\right)\right.$

$\left.\cdot\left(\lambda(y) a \lambda\left(z^{-1} y^{-1} u x\right) \otimes u\right) d u d z d y\right] \xi$

$=\Delta(x) \iint \psi^{*}(y) f(z) \varphi\left(z^{-1} y^{-1} x\right) \lambda(y) a \lambda\left(z^{-1} y^{-1} x\right) \xi d z d y$

$=\Delta(x) \iint \psi^{*}(y) f(z) \lambda(y) a U(\xi \otimes \varphi)\left(z^{-1} y^{-1} x\right) d z d y$

$=\Delta(x) \int \psi^{*}(y) \lambda(y)(a \otimes f) U(\xi \otimes \varphi)\left(y^{-1} x\right) d y$

$=\Delta(x) \delta \circ \lambda\left(\psi^{*}\right)(a \otimes f) U(\xi \otimes \varphi)(x)$.

Furthermore, we have

$$
\begin{aligned}
& \lambda\left(x^{-1}\right) P\left[\delta\left(\lambda(x) \lambda(\psi)^{*}\right)(a \otimes f) \delta \circ \lambda(\varphi)\right] \xi \\
&= P\left[(I \otimes x) \delta \circ \lambda\left(\psi^{*}\right)(a \otimes f) \delta \circ \lambda(\varphi)\right] \xi \\
&= P\left[\iiint \psi^{*}(y) f(z) \varphi(u)(I \otimes x)(\lambda(y) \otimes y)\right. \\
&\cdot(a \otimes z)(\lambda(u) \otimes u) d u d z d y] \xi \quad\left(u \rightarrow z^{-1} y^{-1} x^{-1} u\right) \\
&=P\left[\iiint \psi^{*}(y) f(z) \varphi\left(z^{-1} y^{-1} x^{-1} u\right)\right. \\
&\left.\qquad \cdot\left(\lambda(y) a \lambda\left(z^{-1} y^{-1} x^{-1} u\right) \otimes u\right) d u d z d y\right] \xi \\
&=\iint \psi^{*}(y) f(z) \varphi\left(z^{-1} y^{-1} x^{-1}\right) \lambda(y) a \lambda\left(z^{-1} y^{-1} x^{-1}\right) \xi d z d y \\
&=\iint \psi^{*}(y) f(z) \lambda(y) a U(\xi \otimes \varphi)\left(z^{-1} y^{-1} x^{-1}\right) d z d y \\
&=\int \psi^{*}(y) \lambda(y)(a \otimes f) U(\xi \otimes \varphi)\left(y^{-1} x^{-1}\right) d y \\
&=\delta \circ \lambda\left(\psi^{*}\right)(a \otimes f) U(\xi \otimes \varphi)\left(x^{-1}\right) .
\end{aligned}
$$

So if $g \in C_{00}(G, H)$ we have

$$
\begin{aligned}
\int\left\langle P\left[\delta \circ \lambda\left(\psi^{*}\right)(a \otimes f) \delta\left(\lambda(\varphi) \lambda\left(x^{-1}\right)\right)\right] \lambda(x) \xi, g(x)\right\rangle d x \\
=\int \Delta(x)\left\langle\delta \circ \lambda\left(\psi^{*}\right)(a \otimes f) U(\xi \otimes \varphi)(x), g(x)\right\rangle d x \\
=\left\langle\delta \circ \lambda\left(\psi^{*}\right)(a \otimes f) U(\xi \otimes \varphi), \Delta \cdot g\right\rangle
\end{aligned}
$$


where $\Delta \cdot g(x)=\Delta(x) g(x)$. We also have

$$
\begin{aligned}
\int\left\langle\lambda\left(x^{-1}\right) P[\right. & \left.\left.\delta\left(\lambda(x) \lambda\left(\psi^{*}\right)\right)(a \otimes f) \delta \circ \lambda(\varphi)\right] \xi, g(x)\right\rangle d x \\
& =\int\left\langle\delta \circ \lambda\left(\psi^{*}\right)(a \otimes f) U(\xi \otimes \varphi)\left(x^{-1}\right), g(x)\right\rangle d x \\
& =\left\langle\delta \circ \lambda\left(\psi^{*}\right)(a \otimes f) U(\xi \otimes \varphi), g^{\sim}\right\rangle
\end{aligned}
$$

where $\tilde{g}(x)=\Delta\left(x^{-1}\right) g\left(x^{-1}\right)$.

Now if $a$ is an arbitrary element of $\mathfrak{A}$ we know from the Kaplansky density theorem that there is a net $x_{i} \in \mathfrak{A} \otimes C_{00}(G)$ such that $x_{i} \rightarrow \delta(a)$ in the $\sigma$-topology and $\left\|x_{i}\right\| \leqslant\|\delta(a)\|$. (2.23) and (2.24) together with Corollary 1.3 now gives us that

$$
\begin{gathered}
\int\left\langle P \circ \delta\left(\lambda\left(\psi^{*}\right) a \lambda(\varphi) \lambda\left(x^{-1}\right)\right) \lambda(x) \xi, g(x)\right\rangle d x \\
=\left\langle\delta\left(\lambda\left(\psi^{*}\right) a\right) U(\xi \otimes \varphi), \Delta \cdot g\right\rangle
\end{gathered}
$$

and

$$
\begin{gathered}
\int\left\langle\lambda\left(x^{-1}\right) P \circ \delta\left(\lambda(x) \lambda\left(\psi^{*}\right) a \lambda(\varphi)\right) \xi, g(x)\right\rangle d x \\
=\left\langle\delta\left(\lambda\left(\psi^{*}\right) a\right) U(\xi \otimes \varphi), g^{\tilde{\gamma}}\right\rangle .
\end{gathered}
$$

(Note that if $\left\{x_{i}\right\}$ is a bounded net in $\mathfrak{A}$ converging to $x$ in the $\sigma$-topology, then $\left\langle x_{i}, \varphi\right\rangle \rightarrow\langle x, \varphi\rangle$ uniformly when $\varphi$ is in a norm-compact subset of $\mathfrak{A}_{*}$ ).

Since this shall hold for all $g \in C_{00}(G, H)$ we must have that $(2.21)$ and (2.22) hold locally almost everywhere, so in what follows we can without loss of generality assume that they hold for all $x \in G$.

LEMMA 2.10. If $U$ is as in Lemma 2.9, $f, \varphi \in C_{00}(G)$ and $\xi \in H$, then

$$
\int_{G} f\left(x^{-1}\right) U(\xi \otimes \varphi)(y x) d x=\delta \circ \lambda(\varphi)(\xi \otimes f)(y) .
$$

Proof.

$$
\begin{aligned}
\int_{G} f\left(x^{-1}\right) U(\xi \otimes \varphi)(y x) d x & =\int_{G} f\left(x^{-1}\right) \varphi(y x) \lambda(y x) \xi d x \\
& =\int_{G} \varphi(x) \lambda(x)(\xi \otimes f)\left(x^{-1} y\right) d x \\
& =\int_{G} \varphi(x) \delta \circ \lambda(x)(\xi \otimes f)(y) d x \\
& =\delta \circ \lambda(\varphi)(\xi \otimes f)(y) .
\end{aligned}
$$

So if we for $f \in C_{00}(G)$ define an operator $R_{f}$ over $L^{2}(G, H)$ by

$$
R_{f} g(y)=\int_{G} f\left(x^{-1}\right) g(y x) d x
$$


then $R_{f} \in(\mathfrak{A} \otimes \mathcal{L}(G))^{\prime}$ and

$$
R_{f} U(\xi \otimes \varphi)=\delta \circ \lambda(\varphi)(\xi \otimes f) .
$$

Proposition 2.11. $\mathfrak{A}$ is generated by $\lambda(G)$ and $A$.

Proof. Since $\delta$ is an isomorphism it suffices to prove that $\delta \circ \lambda(G)$ and $\delta(A)$ generates $\delta(\mathfrak{U})$, and this is the same as showing that

$$
B \in \delta \circ \lambda(G)^{\prime} \cap \delta(A)^{\prime} \Rightarrow B \in \delta(\mathfrak{H})^{\prime} .
$$

For this it suffices to prove that $B$ commutes with all elements of the form $\delta\left(\lambda(\psi)^{*} a \lambda(\varphi)\right)$ with $\varphi, \psi \in C_{00}(G), a \in \mathfrak{A}$ since such elements generate $\delta(\mathfrak{A})$. Now let $f, g \in C_{00}(G), \xi, \eta \in H$, then

$$
\begin{aligned}
\left\langleB \delta \left(\lambda(\psi)^{*} a \lambda\right.\right. & (\varphi))(\xi \otimes f), \eta \otimes g\rangle \quad(2.25) \\
& =\left\langle B \delta\left(\lambda\left(\psi^{*}\right) a\right) R_{f} U(\xi \otimes \varphi), \eta \otimes g\right\rangle \\
& =\left\langle R_{f} \delta\left(\lambda\left(\psi^{*}\right) a\right) U(\xi \otimes \varphi), B^{*}(\eta \otimes g)\right\rangle \\
& =\iint f\left(x^{-1}\right)\left\langle\delta\left(\lambda\left(\psi^{*}\right) a\right) U(\xi \otimes \varphi)(y x), B^{*}(\eta \otimes g)(y)\right\rangle d x d y \\
& =\iint f\left(x^{-1} y\right)\left\langle\delta\left(\lambda\left(\psi^{*}\right) a\right) U(\xi \otimes \varphi)(x), B^{*}(\eta \otimes g)(y)\right\rangle d x d y .
\end{aligned}
$$

Fubini holds for this integral since the function $y \rightarrow \int f\left(x^{-1} y\right) g(x) d x$ is square-integrable for any $f \in C_{00}(G), g \in L^{2}(G)$. So changing the order of integration and using (2.21), we get:

$$
\begin{aligned}
& \iint f\left(x^{-1} y\right) \Delta\left(x^{-1}\right)\left\langle P \circ \delta\left(\lambda(\psi)^{*} a \lambda(\varphi) \lambda\left(x^{-1}\right)\right) \lambda(x) \xi, B^{*}(\eta \otimes g)(y)\right\rangle d y d x \\
& =\iint \Delta\left(x^{-1}\right)\left\langle\delta\left[P \circ \delta\left(\lambda(\psi)^{*} a \lambda(\varphi) \lambda\left(x^{-1}\right)\right) \lambda(x)\right]\right. \\
& \left.\cdot(\xi \otimes f)(y), B^{*}(\eta \otimes g)(y)\right\rangle d y d x \\
& =\int \Delta\left(x^{-1}\right)\left\langle\delta\left[P \circ \delta\left(\lambda(\psi)^{*} a \lambda(\varphi) \lambda\left(x^{-1}\right)\right) \lambda(x)\right](\xi \otimes f), B^{*}(\eta \otimes g)\right\rangle d x \\
& =\int\left\langle B(\xi \otimes f), \delta\left[\lambda(x) P \circ \delta\left(\lambda\left(x^{-1}\right) \lambda(\varphi)^{*} a^{*} \lambda(\psi)\right)\right](\eta \otimes g)\right\rangle d x \\
& =\iint\left\langle B(\xi \otimes f)(y), \lambda(x) P \circ \delta\left(\lambda\left(x^{-1}\right) \lambda(\varphi)^{*} a^{*} \lambda(\psi)\right) \eta\right\rangle \overline{g\left(x^{-1} y\right)} d y d x \\
& =\iint\left\langle B(\xi \otimes f)(y), \delta\left(\lambda(\varphi)^{*} a^{*}\right) U(\eta \otimes \psi)(x)\right\rangle \overline{g\left(x^{-1} y\right)} d y d x .
\end{aligned}
$$

By the same reasoning as above we can change the order of integration, so we 
get

$$
\begin{aligned}
& =\iint\left\langle B(\xi \otimes f)(y), g\left(x^{-1} y\right) \delta\left(\lambda(\varphi)^{*} a^{*}\right) U(\eta \otimes \psi)(x)\right\rangle d x d y \\
& =\int\left\langle B(\xi \otimes f)(y), R_{g} \delta\left(\lambda(\varphi)^{*} a^{*}\right) U(\eta \otimes \psi)(y)\right\rangle d y \\
& =\left\langle B(\xi \otimes f), \delta\left(\lambda(\varphi)^{*} a^{*}\right) R_{g} U(\eta \otimes \psi)\right\rangle \\
& =\left\langle B(\xi \otimes f), \delta\left(\lambda(\varphi)^{*} a^{*}\right) \delta \circ \lambda(\psi)(\eta \otimes g)\right\rangle \\
& =\left\langle\delta\left(\lambda(\psi)^{*} a \lambda(\varphi)\right) B(\xi \otimes f), \eta \otimes g\right\rangle .
\end{aligned}
$$

Since this holds for all $f, g \in C_{00}(G), \xi, \eta \in H$ we have that $B \delta\left(\lambda(\psi)^{*} a \lambda(\varphi)\right)$ $=\delta\left(\lambda(\psi)^{*} a \lambda(\varphi)\right) B$.

Proof of Theorem 1. With $A$ and $\rho$ as defined just before Lemma 2.8, we shall prove that $\tilde{\mathfrak{U}}=W_{r}^{*}(A, \rho, G) \cong \mathfrak{A}$. $\tilde{\mathfrak{A}}$ is generated over $L^{2}(G, H)$ by $\tilde{\lambda}(G) \cup \tilde{\rho}(A)$ where $\tilde{\lambda}(x) f(y)=f\left(x^{-1} y\right)$ and $\tilde{\rho}(a) f(y)=\lambda\left(y^{-1}\right) a \lambda(y) f(y)$ for $a \in A, f \in L^{2}(G, H), x, y \in G$. Now let $U$ be the unitary operator over $L^{2}(G, H)$ defined in Lemma 2.9 , then

$$
U \tilde{\lambda}(x) U^{*} f(y)=\lambda(y) U^{*} f\left(x^{-1} y\right)=\lambda(x) f\left(x^{-1} y\right)=\delta \circ \lambda(x) f(y) .
$$

So $U \tilde{\lambda}(x) U^{*}=\delta \circ \lambda(x)$ for $x \in G$.

$$
\begin{aligned}
U \tilde{\rho}(a) U^{*} f(y) & =\lambda(y) \tilde{\rho}(a) U^{*} f(y)=a \lambda(y) U^{*} f(y)=a f(y) \\
& =(a \otimes I) f(y)=\delta(a) f(y) .
\end{aligned}
$$

So $U \tilde{\rho}(a) U^{*}=\delta(a)$ for $a \in A$. Therefore the map $x \rightarrow U x U^{*}$ sets up an isomorphism between $\tilde{\mathfrak{U}}$ and $\delta(\mathfrak{H})$ (the map is onto by Proposition 2.11). Since $\delta$ is an isomorphism, $\tilde{\mathfrak{U}}$ and $\mathfrak{A}$ are isomorphic, and the isomorphism is such that $\tilde{\lambda}(x)$ corresponds to $\lambda(x)$ and the canonical comodule map $\tilde{\delta}$ of $\tilde{\mathfrak{A}}$ corresponds to $\delta$.

We have earlier seen that $\delta$ defines an action of $A(G)$ on $\mathfrak{A}_{*}$ and we proved in Lemma 2.4 that

$$
\left\{\varphi \alpha \mid \varphi \in \mathfrak{A}_{*}, \alpha \in A(G)\right\} \text { spans a norm dense set in } \mathfrak{A}_{*} .
$$

We can now also prove

$$
\text { If } \varphi \alpha=0 \text { for all } \alpha \in A(G) \text {, then } \varphi=0 \text {. }
$$

For actions of *-algebras on Hilbert spaces (2.26) and (2.27) are equivalent, but not in general for actions on Banach spaces. To prove (2.27), suppose $\varphi \in \mathfrak{A}_{*}$ with $\varphi \alpha=0$ for all $\alpha \in A(G)$. Then

$$
\begin{aligned}
0 & =\langle a \lambda(x), \varphi \alpha\rangle=\langle\delta(a \lambda(x)), \varphi \otimes \alpha\rangle=\langle a \lambda(x) \otimes x, \varphi \otimes \alpha\rangle \\
& =\langle a \lambda(x), \varphi\rangle\langle x, \alpha\rangle \text { for all } a \in A, x \in G, \alpha \in A(G) .
\end{aligned}
$$


Since $A(G)$ separates points in $G$, we must have $\langle a \lambda(x), \varphi\rangle=0$ for all $a \in A$, $x \in G$. So from Proposition $2.11\langle a, \varphi\rangle=0$ for all $a \in \mathfrak{A}$, thus $\varphi=0$.

$W^{*}$-crossed products over an abelian group. Suppose now that $G$ is an abelian locally compact group, $\hat{G}$ its dual group, and we shall always assume that the Haar-measures ( $d x$ and $d \gamma$ respectively) are chosen such that the Plancherel theorem holds. Theorem 1 can now be restated as follows:

THEOREM 2. If $\mathfrak{A}$ is a von Neumann algebra and $G$ is a locally compact abelian group, then $\mathfrak{A}$ is isomorphic to a regular $W^{*}$-crossed product $W_{r}^{*}(A, \rho, G)$ for some covariant system $(A, \rho, G)$ over $G$ if and only if there is a $\sigma$-continuous homomorphism $\lambda: G \rightarrow \mathfrak{A}_{u}$ and a $\sigma$-continuous automorphic representation $\tau$ of the dual group $\hat{G}$ such that

$$
\tau_{\gamma}(\lambda(x))=\gamma(x) \lambda(x) \text { for all } x \in G, \gamma \in \hat{G} .
$$

$A$ and $\rho$ are uniquely determined from $\lambda$ and $\tau$ by

$$
\begin{aligned}
A & =\left\{a \in \mathfrak{A} \mid \tau_{\gamma}(a)=\text { a for all } \gamma \in \hat{G}\right\}, \\
\rho_{x}(a) & =\lambda(x) a \lambda\left(x^{-1}\right) \text { for } a \in A, x \in G .
\end{aligned}
$$

Proof. Suppose we start with a $\sigma$-continuous automorphic representation $\rho$ of $G$ over the von Neumann algebra $A$, and $\mathfrak{A}=W_{r}^{*}(A, \rho, G)$ is constructed as described before as a von Neumann algebra over $L^{2}(G, H)$. Define a continuous unitary representation $U$ of $\hat{G}$ by

$$
U_{\gamma} f(x)=\gamma(x) f(x) \text { for } \gamma \in \hat{G}, f \in L^{2}(G, H), x \in G .
$$

Define $\tilde{\rho}$ and $\lambda$ as in (2.1) and (2.2), then it is easy to check that

$$
\begin{aligned}
& U_{\gamma} \tilde{\rho}(a) U_{-\gamma}=\tilde{\rho}(a) \text { for } a \in A, \\
& U_{\gamma} \lambda(x) U_{-\gamma}=\gamma(x) \lambda(x) \text { for } x \in G .
\end{aligned}
$$

So if we define

$$
\tau_{\gamma}(a)=U_{\gamma} a U_{-\gamma} \text { for } a \in \mathfrak{A}, \gamma \in \hat{G},
$$

$\tau$ will be a $\sigma$-continuous automorphic representation of $G$ over $\mathfrak{A}$ such that (2.28) holds. The uniqueness part of Theorem 2, i.e. that $\tilde{\rho}(A)=\left\{a \in \mathfrak{A} \mid \tau_{\gamma}(a)\right.$ $=a$ for all $\gamma \in \hat{G}\}$ will now follow from the uniqueness part of Theorem 1 if we can prove that

$$
W^{*}(a \otimes I) W=a \otimes I \Leftrightarrow \tau_{\gamma}(a)=a \text { for all } \gamma \in G,
$$

where $W$ is the unitary operator over $L^{2}(G \times G, H)$ defined by (2.3).

First let $\Phi$ be the Fourier transform from $L^{2}(G \times G, H)$ to $L^{2}(G \times \hat{G}, H)$ given by

$$
\Phi f(x, \gamma)=\int_{G} \gamma(y) f(x, y) d y \quad \text { for } f \in L^{2}(G \times G, H) .
$$


A straightforward computation shows that

$$
\Phi W^{*} \Phi^{-1} f(x, \gamma)=\gamma(x) f(x, \gamma) \text { for } f \in L^{2}(G \times \hat{G}, H)
$$

so if we define a unitary operator over $L^{2}\left(\hat{G}, L^{2}(G, H)\right)$ by

$$
U f(\gamma)=U_{\gamma} f(\gamma)
$$

and identify $L^{2}\left(\hat{G}, L^{2}(G, H)\right)$ and $L^{2}(G \times \hat{G}, H)$ we have that $\Phi W^{*} \Phi^{-1}=$ $U . \Phi(a \otimes I)=(a \otimes I) \Phi$ for $a \in \mathfrak{A}$, so we have:

$$
\begin{aligned}
W^{*}(a \otimes I) W=a & \otimes I \Leftrightarrow \Phi W^{*} \Phi^{-1}(a \otimes I) \Phi W \Phi^{-1}=a \otimes I \\
& \Leftrightarrow U(a \otimes I) U^{*}=a \otimes I \Leftrightarrow U_{\gamma} a U_{-\gamma}=a \text { for all } \gamma \in \hat{G} .
\end{aligned}
$$

This proves (2.30), and the uniqueness of Theorem 2 follows.

Let us now turn to the existence part of the theorem, so suppose we have a von Neumann algebra $\mathfrak{A}$ and maps $\lambda: G \rightarrow \mathfrak{A}_{u}, \tau: \hat{G} \rightarrow$ Aut(2) such that (2.28) holds. We are going to use Theorem 1 , so we will have to construct a map $\delta: \mathfrak{U} \rightarrow \mathfrak{A} \otimes \mathfrak{L}(G)$ with the properties in Theorem 1 . We first observe that $\mathcal{L}(G) \cong L^{\infty}(\hat{G})$ as von Neumann algebras with $x \in G$ corresponding to the function $\hat{x}: \gamma \rightarrow \gamma(x)$ in $L^{\infty}(\hat{G})$. Since $\mathfrak{A} \otimes L^{\infty}(\hat{G}) \cong L^{\infty}(\hat{G}, \mathfrak{A})$ it therefore suffices to get a map $\delta: \mathfrak{A} \rightarrow L^{\infty}(\hat{G}, \mathfrak{A})$, and this is simply done by defining

$$
\delta(a)(\gamma)=\tau_{\gamma}(a) \text { for } a \in \mathfrak{A}, \gamma \in \hat{G} .
$$

$\delta$ is then a normal *-isomorphism from $\mathfrak{A}$ into $L^{\infty}(\hat{G}, \mathfrak{A})$.

$$
\begin{array}{r}
\delta(\lambda(x))(\gamma)=\tau_{\gamma}(\lambda(x))=\gamma(x) \lambda(x)=(\lambda(x) \otimes \hat{x})(\gamma) \\
\text { for } x \in G, \gamma \in \hat{G},
\end{array}
$$

so $\delta \circ \lambda(x)=\lambda(x) \otimes x$.

When we identify $\mathscr{L}(G)$ with $L^{\infty}(\hat{G})$ and $\mathscr{L}(G) \otimes \mathfrak{L}(G)$ with $L^{\infty}(\hat{G} \times \hat{G})$, the map $\delta_{G}: \mathcal{L}(G) \rightarrow \mathcal{L}(G) \otimes \mathcal{L}(G)$ corresponds to the map $\delta_{G}: L^{\infty}(\hat{G}) \rightarrow$ $L^{\infty}(\hat{G} \times \hat{G})$ given by

$$
\delta_{G} \varphi(\alpha, \beta)=\varphi(\alpha+\beta) .
$$

In fact, if $x \in G$ we have

$$
\delta_{G}(x)^{\wedge}(\alpha, \beta)=\hat{x} \otimes \hat{x}(\alpha, \beta)=\alpha(x) \beta(x)=(\alpha+\beta)(x)=\hat{x}(\alpha+\beta) .
$$

Since $L^{\infty}(\hat{G})$ is generated by $\{\hat{x} \mid x \in G\}$, (2.31) holds for all $\varphi \in L^{\infty}(\hat{G})$.

Finally we want to check the formula $(\delta \otimes i) \delta(a)=\left(i \otimes \delta_{G}\right) \delta(a)$ for $a \in \mathfrak{A}$, where both sides are considered as elements in $L^{\infty}(\hat{G} \times \hat{G}, \mathfrak{A})$ :

$$
\begin{aligned}
& (\delta \otimes i) \delta(a)(\alpha, \beta)=\tau_{\alpha}(\delta(a)(\beta))=\tau_{\alpha} \tau_{\beta}(a)=\tau_{\alpha+\beta}(a) \\
& =\delta(a)(\alpha+\beta)=\left(i \otimes \delta_{G}\right) \delta(a)(\alpha, \beta) \text { for } \alpha, \beta \in \hat{G} .
\end{aligned}
$$

So $(\delta \otimes i) \delta(a)=\left(i \otimes \delta_{G}\right) \delta(a)$ for all $a \in \mathfrak{A}$, and the triple $(\mathfrak{A}, \lambda, \delta)$ satisfies 
the assumptions in Theorem 1 . Hence $\mathfrak{A} \cong W_{r}^{*}(A, \rho, G)$ where $A=\{a \in \mathfrak{A} \mid \delta(a)=a \otimes I\}$ and $\rho_{x}(a)=\lambda(x) a \lambda\left(x^{-1}\right)$ for $a \in G, x \in G$.

$$
\delta(a)(\alpha)=(a \otimes I)(\alpha) \Leftrightarrow \tau_{\alpha}(a)=a \text { for } \alpha \in \hat{G},
$$

so $\delta(a)=a \otimes I \Leftrightarrow \tau_{\alpha}(a)=a$ for all $\alpha \in \hat{G}$, thus $A=\left\{a \in \mathfrak{A} \mid \tau_{\alpha}(a)=a\right.$ for all $\alpha \in \hat{G}\}$.

Theorem 2 tells us that a covariant system $(A, \rho, G)$ over an abelian group $G$ naturally gives rise to a new covariant system $(\mathfrak{A}, \tau, \hat{G})$ over $\hat{G}$, where $\mathfrak{A}=W_{r}^{*}(A, \rho, G)$. ( $(\mathfrak{A}, \tau, \hat{G})$ then gives us a third covariant system $\left(W_{r}^{*}(\mathfrak{A}, \tau, \hat{G}), \hat{\rho}, G\right)$ and it is natural to ask what relation this covariant system has to $(A, \rho, G)$. This question has been answered by M. Takesaki in [28, Theorems 4.5 and 4.6]: $W_{r}^{*}(\mathfrak{A}, \tau, \hat{G}) \cong A \otimes \mathscr{B}\left(L^{2}(G)\right)$ and under this isomorphism $\hat{\rho}_{x}$ corresponds to $\rho_{x} \otimes \mathrm{ad}(-x)$ where ad is the automorphic representation of $G$ on $\Re\left(L^{2}(G)\right)$ defined by $\operatorname{ad}(x) a=x a x^{-1}$ for $x \in G$, $a \in \mathscr{B}\left(L^{2}(G)\right)$.

These facts have been used by M. Takesaki to show that all type III von Neumann algebras can be obtained as a regular $W^{*}$-crossed product $W_{r}^{*}(\mathfrak{A}, \rho, \mathbf{R})$ over the real numbers with $\mathfrak{U}$ a properly infinite, semifinite von Neumann algebra, cf. [28] and the more special results in [27].

\section{Chapter 3. The Duality Theorem for $C^{*}$-Crossed Products}

Let $A$ be a $C^{*}$-algebra over a Hilbert space $H$, and let $\rho$ be a normcontinuous representation of a locally compact group $G$ over $A$, i.e. $\rho$ is a homomorphism from $G$ into the group $\operatorname{Aut}(A)$ such that the map $x \rightarrow \rho_{x}(a)$ from $G$ to $A$ is norm-continuous for each $a \in A$. We can then form a new $C^{*}$-algebra $C^{*}(A, \rho, G)$ called the $C^{*}$-crossed product of $A$ and $G$ or the $C^{*}$-covariance algebra of $(A, \rho, G)$ as follows:

In $C_{00}(G, A)$ we define a convolution, an involution and a norm by

$$
\begin{aligned}
\varphi \psi(x) & =\int_{G} \varphi(y) \rho_{y}\left(\psi\left(y^{-1} x\right)\right) d y, \\
\varphi^{*}(x) & =\Delta\left(x^{-1}\right) \rho_{x}\left(\varphi\left(x^{-1}\right)^{*}\right), \\
\|\varphi\| & =\int_{G}\|\varphi(x)\| d x
\end{aligned}
$$

for $\varphi, \psi \in C_{00}(G, A) . C_{00}(G, A)$ then becomes a normed *-algebra, and $C^{*}(A, \rho, G)$ is simply the enveloping $C^{*}$-algebra of $C_{00}(G, A)(\mathrm{cf} .[9,2.7 .2])$. $C^{*}(A, \rho, G)$ has a regular representation $\tilde{\rho}$ over $X=H \otimes L^{2}(G) \cong L^{2}(G, H)$ given by

$$
\begin{aligned}
& \tilde{\rho}(\varphi) f(x)=\int \rho_{x^{-1}}(\varphi(y)) f\left(y^{-1} x\right) d y \\
& \qquad \text { for } \varphi \in C_{00}(G, A), f \in L^{2}(G, H) .
\end{aligned}
$$


It is easy to see that $\tilde{\rho}$ is a bounded *-representation of $C_{00}(G, A)$, so $\tilde{\rho}$ has a unique extension to a ${ }^{*}$-representation of $C^{*}(A, \rho, G)$. Let $C_{r}^{*}(A, \rho, G)=$ $\tilde{\rho}\left(C^{*}(A, \rho, G)\right)$, this $C^{*}$-algebra is called the regular $C^{*}$-crossed product of $G$ and $A$ or the regular covariance algebra of $(A, \rho, G)$.

As in Chapter 2 one can check that this definition of $C_{r}^{*}(A, \rho, G)$ does not depend on the Hilbert space $H$ on which $A$ is represented.

The analogue of Lemma 2.1 in this situation is as follows: $\rho$ is said to be inner if there is a homomorphism $U$ from $G$ into the unitary group of $M(A)$ such that $U$ is continuous in the multiplier-topology and $\rho_{x}(a)=U_{x} a U_{x^{-1}}$ for all $a \in A, x \in G$. Then $C_{r}^{*}(A, \rho, G)=U^{*}\left(A \otimes C_{r}^{*}(G)\right) U$ where $U$ is the unitary operator defined in Lemma 2.1. The proof also goes as indicated there, one should only note that $C_{r}^{*}(A, \rho, G)$ is generated by the set

$$
\left\{U^{*}(a \otimes f) U \mid a \in A, f \in C_{00}(G)\right\} .
$$

If $G$ is an amenable group we will have that $C_{r}^{*}(A, \rho, G) \cong C^{*}(A, \rho, G)$, cf. [33, Proposition 2.2]. If $\lambda$ is the continuous representation of $G$ over $L^{2}(G, H)$ given by

$$
\lambda(x) f(y)=f\left(x^{-1} y\right) \text { for } f \in L^{2}(G, H), x, y \in G,
$$

it is easy to see that

$$
\lambda(x) \tilde{\rho}\left(C_{00}(G, A)\right) \subseteq \tilde{\rho}\left(C_{00}(G, A)\right)
$$

and

$$
\tilde{\rho}\left(C_{00}(G, A)\right) \lambda(x) \subseteq \tilde{\rho}\left(C_{00}(G, A)\right) .
$$

Therefore $\lambda(x) \in M\left(C_{r}^{*}(A, \rho, G)\right)$, and since the map $x \rightarrow \lambda(x) \tilde{\rho}(\varphi)$ will be norm-continuous for $\varphi \in C_{00}(G, A), \lambda$ is a continuous map from $G$ into $M\left(C_{r}^{*}(A, \rho, G)\right)$ with the multiplier topology, i.e. the map $x \rightarrow \lambda(x) a$ is continuous for all $a \in C_{r}^{*}(A, \rho, G)$.

We can also define a *-isomorphism of $A$ over $L^{2}(G, H)$ by

$$
\tilde{\rho}(a) f(x)=\rho_{x^{-1}}(a) f(x) \quad \text { for } a \in A, f \in L^{2}(G, H) .
$$

We will have $\tilde{\rho}(a) \tilde{\rho}\left(C_{00}(G, A)\right) \subseteq \tilde{\rho}\left(C_{00}(G, A)\right)$ and $\tilde{\rho}\left(C_{00}(G, A)\right) \tilde{\rho}(a) \subseteq$ $\tilde{\rho}\left(C_{00}(G, A)\right)$. Thus $\tilde{\rho}(a) \in M\left(C_{r}^{*}(A, \rho, G)\right)$, and $\tilde{\rho}$ is an isomorphism of $A$ into $M\left(C_{r}^{*}(A, \rho, G)\right)$.

As in Chapter 2 we shall define a unitary operator $W$ over $X \otimes L^{2}(G) \cong$ $L^{2}(G \times G, H)$ by

$$
W f(s, t)=f(s, s t) \text { for } f \in L^{2}(G \times G, H), s, t \in G .
$$


Then we will have for $\varphi \in C_{00}(G, A), f \in L^{2}(G \times G, H)$ that

$$
\begin{aligned}
W^{*}(\tilde{\rho}(\varphi) \otimes I) W f(s, t) & =(\tilde{\rho}(\varphi) \otimes I) W f\left(s, s^{-1} t\right) \\
& =\int_{G} \rho_{s^{-1}}(\varphi(y)) W f\left(y^{-1} s, s^{-1} t\right) d y \\
& =\int_{G} \rho_{s^{-1}}(\varphi(y)) f\left(y^{-1} s, y^{-1} t\right) d y .
\end{aligned}
$$

Let $C_{r}^{*}(G)$ denote the $C^{*}$-algebra generated by the left regular representation of $C_{00}(G)$ over $L^{2}(G)$, and define the map $\delta$ from $C_{r}^{*}(A, \rho, G)$ by

$$
\delta(a)=W^{*}(a \otimes I) W \text { for } a \in C_{r}^{*}(A, \rho, \dot{G}) .
$$

So $\delta$ is defined exactly as in Chapter 2. $\delta$ is a ${ }^{*}$-isomorphism, and $\delta\left(C_{r}^{*}(A, \rho, G)\right) \subseteq M\left(C_{r}^{*}(A, \rho, G) \otimes C_{r}^{*}(G)\right)$. In fact, if $\varphi, \psi \in C_{00}(G, A), \alpha$ $\in C_{00}(G), f \in L^{2}(G \times G, H)$ :

$$
\begin{aligned}
\delta(\tilde{\rho}(\varphi))(\tilde{\rho}(\psi) & \otimes \alpha) f(s, t) \\
= & \int_{G} \rho_{s^{-1}}(\varphi(y))(\tilde{\rho}(\psi) \otimes \alpha) f\left(y^{-1} s, y^{-1} t\right) d y \\
= & \iiint \rho_{s^{-1}}(\varphi(y)) \rho_{s^{-1} y}(\psi(z)) \alpha(x) f\left(z^{-1} y^{-1} s, x^{-1} y^{-1} t\right) d x d z d y \\
& \left(x \rightarrow y^{-1} x, z \rightarrow y^{-1} z\right) \\
= & \iint_{G} \rho_{s^{-1}}\left(\int_{G} \alpha\left(y^{-1} x\right) \varphi(y) \rho_{y}\left(\psi\left(y^{-1} z\right)\right) d y\right) f\left(z^{-1} s, x^{-1} t\right) d z d x .
\end{aligned}
$$

From this we see that $\delta(\tilde{\rho}(\varphi))(\tilde{\rho}(\psi) \otimes \alpha) \in C_{r}^{*}(A, \rho, G) \otimes C_{r}^{*}(G)$ and similarly that $(\tilde{\rho}(\psi) \otimes \alpha) \delta(\tilde{\rho}(\varphi)) \in C_{r}^{*}(A, \rho, G) \otimes C_{r}^{*}(G)$. It will follow that $\delta(\tilde{\rho}(\varphi)) \in M\left(C_{r}^{*}(A, \rho, G) \otimes C_{r}^{*}(G)\right)$ for all $\varphi \in C_{00}(G, A)$, hence $\delta\left(C_{r}^{*}(A, \rho, G)\right) \subseteq M\left(C_{r}^{*}(A, \rho, G) \otimes C_{r}^{*}(G)\right)$. Furthermore if $\varphi \in C_{00}(G, A)$, $\alpha \in C_{00}(G), f \in L^{2}(G \times G, H)$,

$$
\begin{aligned}
\delta(\tilde{\rho}(\varphi))(I & \otimes \alpha) f(s, t) \\
& =\int_{G} \int_{G} \rho_{s^{-1}}(\varphi(y)) \alpha(x) f\left(y^{-1} s, x^{-1} y^{-1} t\right) d x d y \quad\left(x \rightarrow y^{-1} x\right) \\
& =\int_{G} \int_{G} \alpha\left(y^{-1} x\right) \rho_{s^{-1}}(\varphi(y)) f\left(y^{-1} s, x^{-1} t\right) d x d y .
\end{aligned}
$$

So $\delta(\tilde{\rho}(\varphi))(I \otimes \alpha) \in C_{r}^{*}(A, \rho, G) \otimes C_{r}^{*}(G)$ and similarly $(I \otimes \alpha) \delta(\tilde{\rho}(\varphi)) \in$ $C_{r}^{*}(A, \rho, G) \otimes C_{r}^{*}(G)$. From this we see that $(I \otimes \alpha) \delta(a)$ and $\delta(a)(I \otimes \alpha)$ are in $C_{r}^{*}(A, \rho, G) \otimes C_{r}^{*}(G)$ for all $a \in C_{r}^{*}(A, \rho, G)$ and $\alpha \in C_{r}^{*}(G)$. So $\delta$ is a ${ }^{*}$-isomorphism of $C_{r}^{*}(A, \rho, G)$ into $\tilde{M}\left(C_{r}^{*}(A, \rho, G) \otimes C_{r}^{*}(G)\right.$ ). (Recall that if $\mathfrak{A}$ and $\mathfrak{B}$ are $C^{*}$-algebras, $\tilde{M}(\mathfrak{U} \otimes \mathfrak{B})=\{x \in M(\mathfrak{A} \otimes \mathfrak{B}) \mid x(I \otimes \mathfrak{B})+$ $(I \otimes \mathfrak{B}) x \subseteq \mathfrak{U} \otimes \mathfrak{B}\}$.) (3.2) also defines $\delta$ as a ${ }^{*}$-isomorphism of 
$M\left(C_{r}^{*}(A, \rho, G)\right)$ and as in Chapter 2

$$
\begin{array}{ll}
\delta(\tilde{\rho}(a))=\tilde{\rho}(a) \otimes I & \text { for } a \in A, \\
\delta(\lambda(x))=\lambda(x) \otimes x & \text { for } x \in G .
\end{array}
$$

If $\delta_{G}$ is the restriction to $C_{r}^{*}(G)$ of the map defined on $\mathcal{L}(G)$ in Chapter 2 we of course have

$$
(\delta \otimes i) \delta(a)=\left(i \otimes \delta_{G}\right) \delta(a) \text { for } a \in C_{r}^{*}(A, \rho, G) .
$$

A map $\delta$ satisfying (3.5) is called nondegenerate if for every $\varphi \in \mathfrak{U}^{*} \backslash\{0\}$ there is an $\alpha \in C_{r}^{*}(G)^{*}$ with $(\varphi \otimes \alpha) \circ \delta \neq 0$. (Note that $\varphi \otimes \alpha$ has a unique extension to a linear functional on $M\left(\mathscr{A} \otimes C_{r}^{*}(G)\right)$ continuous in the multiplier topology.) If $\delta$ is as defined in (3.2) it is easy to check that $\delta$ is nondegenerate. The duality theorem for $C^{*}$-crossed products can now be stated as follows:

THeOREM 3. If $\mathfrak{A}$ is a $C^{*}$-algebra and $G$ is a locally compact group, $\mathfrak{A}$ is isomorphic to a regular $C^{*}$-crossed product $C_{r}^{*}(A, \rho, G)$ for a norm-continuous automorphic representation $\rho$ of $G$ over a $C^{*}$-algebra $A$ if and only if there is a homomorphism $\lambda: G \rightarrow M(\mathfrak{U})_{u}$ continuous in the multiplier topology on $M(\mathfrak{A})$ and a nondegenerate ${ }^{*}$-isomorphism $\delta: \mathfrak{A} \rightarrow \tilde{M}\left(\mathfrak{A} \otimes C_{r}^{*}(G)\right)$ such that

$$
\begin{aligned}
\delta(\lambda(x)) & =\lambda(x) \otimes x & & \text { for } x \in G, \\
(\delta, \otimes i) \delta(a) & =\left(i \otimes \delta_{G}\right) \delta(a) & & \text { for } a \in \mathfrak{A} .
\end{aligned}
$$

$A$ and $\rho$ are uniquely determined by $\delta$ and $\lambda: A$ consists of all elements $a \in M(\mathfrak{A})$ satisfying

$$
\begin{gathered}
\delta(a)=a \otimes I, \\
a \lambda(f) \text { and } \lambda(f) a \in \mathfrak{U} \text { for all } f \in C_{00}(G), \\
x \rightarrow \lambda(x) a \lambda\left(x^{-1}\right) \text { is norm-continuous. }
\end{gathered}
$$

$\rho$ is defined by $\rho_{x}(a)=\lambda(x) a \lambda\left(x^{-1}\right)$ for $a \in A, x \in G$. If $G$ is amenable or discrete $\delta$ is automatically nondegenerate.

REMARK. In stating this theorem we have already made use of the fact that $\delta$ extends to a ${ }^{*}$-isomorphism of $M(\mathfrak{U})$.

In order to prove the uniqueness of Theorem 3 we shall need the following:

Lemma 3.1. Suppose $A$ is a $C^{*}$-subalgebra of $B$ and that $\rho: G \rightarrow \operatorname{Aut}(B)$ is a norm-continuous automorphic representation of $G$ with $\rho_{x}(A)=A$ for all $x \in$ $G$. Then $C_{r}^{*}(A, \rho, G)$ is a $C^{*}$-subalgebra of $C_{r}^{*}(B, \rho, G)$, and they coincide if and only if $A=B$.

Proof. Take $\mathfrak{A}=C_{r}^{*}(A, \rho, G)$ and $\mathfrak{B}=C_{r}^{*}(B, \rho, G)$, then $\mathfrak{A}$ obviously is a $C^{*}$-subalgebra of $\mathfrak{B}$. We want to prove that $A \neq B \Rightarrow \mathfrak{A} \neq \mathfrak{B}$. Without loss of generality we may assume that $B$ acts on a Hilbert space $H$ such that any 
$\varphi \in B^{*}$ can be realized as $\varphi(b)=\langle b \xi, \eta\rangle$ for suitable vectors $\xi, \eta \in H$. So if $b \in B \backslash A$ there are vectors $\xi, \eta \in H$ such that $\langle b \xi, \eta\rangle \neq 0$ but $\langle a \xi, \eta\rangle=0$ for $a \in A$. Let $U$ be a neighbourhood of $e$ in $G$ such that $\left\langle\rho_{x^{-1}}(b) \xi, \eta\right\rangle \neq 0$ for $x \in U$. Now take $f, \alpha, \beta \in C_{00}(G)$ such that $\alpha$ and $\beta$ both have support in $U, \alpha \geqslant 0, \alpha \neq 0$ and $\int_{G} \overline{\beta(x)}\left\langle\rho_{x^{-1}}(b) \xi, \eta\right\rangle d x \neq 0 . f$ is chosen such that $f(x)=1$ for $x \in U U^{-1}$. For any $\varphi \in C_{00}(G, A)$ we have

$$
\begin{aligned}
\langle\tilde{\rho}(\varphi)(\xi \otimes \alpha), \eta \otimes \beta\rangle & =\int_{G} \int_{G}\left\langle\rho_{x^{-1}}(\varphi(y))(\xi \otimes \alpha)\left(y^{-1} x\right), \eta \otimes \beta(x)\right\rangle d x d y \\
& =\int_{G} \int_{G} \alpha\left(y^{-1} x\right) \overline{\beta(x)}\left\langle\rho_{x^{-1}}(\varphi(y)) \xi, \eta\right\rangle d x d y=0 .
\end{aligned}
$$

So $\langle a(\xi \otimes \alpha), \eta \otimes \beta\rangle=0$ for all $a \in \mathfrak{A}$. Define $\psi \in C_{00}(G, B)$, by $\psi(x)=$ $f(x) b$, then

$$
\begin{aligned}
\langle\tilde{\rho}(\psi)(\xi \otimes \alpha), \eta \otimes \beta\rangle & =\int_{G} \int_{G} \alpha\left(y^{-1} x\right) \overline{\beta(x)} f(y)\left\langle\rho_{x^{-1}}(b) \xi, \eta\right\rangle d y d x \\
& =\int_{G} \int_{G} \alpha\left(y^{-1}\right) f(x y) \overline{\beta(x)}\left\langle\rho_{x^{-1}}(b) \xi, \eta\right\rangle d y d x \\
& =\int_{G} \alpha\left(y^{-1}\right) d y \int_{G} \overline{\beta(x)}\left\langle\rho_{x^{-1}}(b) \xi, \eta\right\rangle d x \neq 0
\end{aligned}
$$

since $x \in U, y^{-1} \in U \Rightarrow x y \in U U^{-1} \Rightarrow f(x y)=1$. So $\tilde{\rho}(\psi) \in \mathscr{B}$,

$$
\langle\tilde{\rho}(\psi)(\xi \otimes \alpha), \eta \otimes \beta\rangle \neq 0,
$$

thus $\tilde{\rho}(\psi) \notin \mathfrak{A}$ and $\mathfrak{A} \neq \mathscr{B}$.

Proof of the uniqueness part of Theorem 3. Suppose we have a normcontinuous automorphic representation $\rho$ of $G$ over a $C^{*}$-algebra $A$ and that $\mathfrak{A}=C_{r}^{*}(A, \rho, G), \lambda$ and $\delta$ is constructed as above. Take $B=\{a \in M(\mathfrak{A}) \mid$ (3.6), (3.7) and (3.8) hold \}, then $\tilde{\rho}(A)$ is a $C^{*}$-subalgebra of $B$, and if we define $\rho^{\prime}: G \rightarrow \operatorname{Aut}(B)$ by $\rho_{x}^{\prime}(b)=\lambda(x) b \lambda\left(x^{-1}\right)$ we have that $\rho^{\prime}$ is a normcontinuous automorphic representation of $G$. If $a \in A, \rho_{x}^{\prime}(\tilde{\rho}(a))=\tilde{\rho}\left(\rho_{x}(a)\right)$ for all $x \in G$, so $\tilde{\rho}(A)$ and $B$ satisfies the assumptions in Lemma 3.1. So to prove that $B=\tilde{\rho}(A)$ it suffices to show that $\mathfrak{B}=C_{r}^{*}\left(B, \rho^{\prime}, G\right)$ and $\tilde{\mathfrak{U}}=$ $C_{r}^{*}\left(\tilde{\rho}(A), \rho^{\prime}, G\right)$ coincide. Take $X=L^{2}(G, H)$, then $\tilde{\mathfrak{U}}$ and $\mathscr{B}$ act on $L^{2}(G, X)$. Define a unitary operator $U$ over $L^{2}(G, X)$ by $U f(x)=\lambda(x) f(x)$ for $f \in L^{2}(G, X)$. For $\alpha \in C_{00}(G, B), \varphi \in L^{2}(G, X)$ define

$$
\tilde{\tilde{\rho}}(\alpha) \varphi(x)=\int_{G} \rho_{x^{-1}}^{\prime}(\alpha(y)) \varphi\left(y^{-1} x\right) d y,
$$

and if $b \in B, f \in C_{00}(G)$ define $b \otimes f \in C_{00}(G, B)$ by $(b \otimes f)(x)=f(x) b$. $\mathfrak{B}$ is then generated by $\left\{\tilde{\tilde{\rho}}(b \otimes f) \mid b \in B, f \in C_{00}(G)\right\}$. We will have that for 
$b \in B, f \in C_{00}(G), \varphi \in L^{2}(G, X):$

$$
\begin{aligned}
U \tilde{\tilde{\rho}}(b & \otimes f) U^{*} \varphi(x)=\int_{G} \lambda(x) \rho_{x^{-1}}^{\prime}(b \otimes f(y)) U^{*} \varphi\left(y^{-1} x\right) d y \\
& =\int_{G} f(y) b \lambda(y) \varphi\left(y^{-1} x\right) d y=b \delta \circ \lambda(f) \varphi(x)=(b \otimes I) \delta \circ \lambda(f) \varphi(x) .
\end{aligned}
$$

So $U \tilde{\tilde{\rho}}(b \otimes f) U^{*}=(b \otimes I) \delta \circ \lambda(f)=\delta(b \lambda(f)) \in \delta(\mathfrak{A})$ since $b \lambda(f) \in \mathfrak{A}$ for $b \in B$ and $f \in C_{00}(G)$. Therefore $U \mathscr{B} U^{*} \subseteq \delta(\mathfrak{A})$. $\tilde{\mathfrak{U}}$ is generated by

$$
\left\{\tilde{\tilde{\rho}}(\tilde{\rho}(a) \otimes f) \mid a \in A, f \in C_{00}(G)\right\},
$$

and $U \tilde{\tilde{\rho}}(\tilde{\rho}(a) \otimes f) U^{*}=\delta(\tilde{\rho}(a) \lambda(f))$. Since $\mathfrak{A}$ is generated by $\{\tilde{\rho}(a) \lambda(f) \mid a \in A$, $\left.f \in C_{00}(G)\right\}, U \tilde{\mathfrak{A}} U^{*}=\delta(\mathfrak{H}) \supseteq U \mathfrak{B} U^{*}$, thus $\mathfrak{B}=\tilde{\mathfrak{A}}$. So from Lemma 3.1 it follows that $\tilde{\rho}(A)=B$. Since the covariant systems $(A, \rho, G)$ and $\left(\tilde{\rho}(A), \rho^{\prime}, G\right)$ are equivalent, the uniqueness in Theorem 3 now follows.

Let us now turn to the existence part of Theorem 3, so we shall suppose we have a $C^{*}$-algebra $\mathfrak{A}$, a nondegenerate ${ }^{*}$-isomorphism $\delta: \mathfrak{A} \rightarrow \tilde{M}(\mathfrak{H} \otimes$ $C_{r}^{*}(G)$ ), and a continuous homomorphism $\lambda: G \rightarrow M(\mathfrak{A})_{u}$ such that (3.4) and (3.5) hold. If we consider $\mathfrak{A}$ over the Hilbert space $H$ given by its universal representation $\mathfrak{A}^{* *} \cong \mathfrak{A}^{\prime \prime}$. By $[9$, Proposition 12.1.5], $\delta$ extends to a normal homomorphism from the von Neumann algebra $\mathfrak{A}^{* *}$ into $\tilde{M}\left(\mathfrak{U} \otimes C_{r}^{*}(G)\right)^{\prime \prime}=$ $\mathfrak{U}^{\prime \prime} \otimes \mathfrak{L}(G)=\mathfrak{A}^{* *} \otimes \mathfrak{L}(G)$, but the extension is not necessarily faithful. Furthermore $\lambda(x) \in \mathfrak{U}^{* *}$. This means that we can use the results in Chapter 2 which do not require that $\delta$ is an isomorphism of $\mathfrak{A}^{* *}$.

Let $B(G)=C^{*}(G)^{*}, B_{r}(G)=C_{r}^{*}(G)^{*}$ then it is known that $B(G)$ is the linear span of all continuous positive definite functions on $G, B_{r}(G)$ is the linear span of the positive definite functions in $B_{r}(G)$, and each positive definite $\varphi \in B_{r}(G)$ can be approximated uniformly on compacta by positive definite functions from $C_{00}(G)$, cf. [9, Proposition 18.3.5]. $A(G) \subset B_{r}(G) \subset$ $B(G)$ with both $A(G)$ and $B_{r}(G)$ norm-closed ideals in $B(G), A(G)$ is $\sigma\left(B_{r}(G), C_{r}^{*}(G)\right)$-dense in $B_{r}(G)$ and $A(G) \cap C_{00}(G)$ is norm-dense in $A(G)$.

As in Chapter 2 we can define an action of $B_{r}(G)$ on $\mathfrak{A}^{*}$ by $\langle a, \varphi \alpha\rangle=$ $\langle\delta(a), \varphi \otimes \alpha\rangle$ for $a \in \mathfrak{A}, \varphi \in \mathfrak{A}^{*}, \alpha \in B_{r}(G)$, where $\varphi \otimes \alpha$ is the unique extension of $\varphi \otimes \alpha$ to $M\left(\mathfrak{A} \otimes C_{r}^{*}(G)\right)$ continuous in the multiplier topology. By almost repeating the proof of Lemma 2.4 one can prove that

$$
\text { linspan }\left\{\varphi \alpha \mid \varphi \in \mathfrak{U}^{*}, \alpha \in B_{r}(G)\right\} \text { is } \sigma\left(\mathfrak{U}^{*}, \mathfrak{U}\right) \text {-dense in } \mathfrak{U}^{*} \text {. }
$$

Using the same notation as in Chapter 2, let

$$
\begin{aligned}
\left(\mathfrak{A} \otimes C_{r}^{*}(G)\right)_{\mathrm{tr}}^{+} & =M\left(\mathfrak{U} \otimes C_{r}^{*}(G)\right) \cap\left(\mathfrak{U}^{* *} \otimes \mathcal{L}(G)\right)_{\mathrm{tr}}^{+}, \\
\mathfrak{J}_{\mathrm{tr}}^{0} & =\mathfrak{\Im}_{\mathrm{tr}} \cap M\left(\mathfrak{A} \otimes C_{r}^{*}(G)\right), \\
\left(\mathfrak{A} \otimes C_{r}^{*}(G)\right)_{\mathrm{tr}} & =\operatorname{linspan}\left(\mathfrak{U} \otimes C_{r}^{*}(G)\right)_{\mathrm{tr}}^{+}=\left(\mathfrak{S}_{\mathrm{tr}}^{0}\right) * \mathfrak{J}_{\mathrm{tr}}^{0} .
\end{aligned}
$$


So for instance $\mathfrak{S}_{\mathrm{tr}}^{0}=\left\{x \in M\left(\mathfrak{A} \otimes C_{r}^{*}(G)\right) \mid \exists K_{x} \geqslant 0, \varphi \otimes \operatorname{tr}\left(x^{*} x\right) \leqslant\right.$ $K_{x}\|\varphi\|$ for all $\left.\varphi \in \mathfrak{U}^{*+}\right\}$. Take $\mathfrak{A}_{0}=\left\{a \in \mathfrak{A} \mid \delta(a) \in \mathfrak{S}_{\mathrm{tr}}^{0}\right\}, \mathfrak{A}_{1}=\{a \in \mathfrak{A} \mid \delta(a)$ $\left.\in\left(\mathfrak{A} \otimes C_{r}^{*}(G)\right)_{\mathrm{tr}}\right\}$ so $\mathfrak{A}_{1}=\mathfrak{U}_{0}^{*} \mathfrak{U}_{0}$. Let $P:\left(\mathfrak{U}^{* *} \otimes \mathcal{L}(G)\right)_{\mathrm{tr}} \rightarrow \mathfrak{U}^{* *}$ be the linear map associated with the Plancherel weight tr defined in Chapter 2. In Lemma 2.6 we proved that $f \in C_{00}(G) \Rightarrow \delta \circ \lambda(f) \in \mathfrak{S}_{\mathrm{tr}}^{0}$ and $P \circ \delta \circ \lambda(f)=f^{*} f(e)=$ $\|f\|_{2}^{2}$. From this we see that $a \lambda(f) \in \mathfrak{A}_{0}$ for $a \in \mathfrak{A}, f \in C_{00}(G)$ and

$$
\left\|P \circ \delta\left(\lambda(f)^{*} a^{*} a \lambda(f)\right)\right\| \leqslant\left\|a^{*} a\right\|\left\|P \circ \delta \circ \lambda\left(f^{*} f\right)\right\|=\|a\|^{2}\|f\|_{2}^{2} .
$$

From this it follows that

$$
\left\|P \circ \delta\left(\lambda(f)^{*} a^{*} a \lambda(g)\right)\right\| \leqslant\|a\|^{2}\|f\|_{2}\|g\|_{2} \text { for } a \in \mathfrak{A}, f, g \in C_{00}(G) \text {. }
$$

The next four lemmas will give as a result that for $f, g \in C_{00}(G), a \in \mathfrak{A}$ we will have $P \circ \delta\left(\lambda(f)^{*} a \lambda(g)\right) \in A=\{a \in M(\mathfrak{U}) \mid$ (3.6), (3.7) and (3.8) hold $\}$.

Lemma 3.2. For $f \in C_{00}(G), a \in \mathfrak{A}$ the map $x \rightarrow P \circ \delta\left(\lambda(f)^{*} a^{*} a \lambda(f) \lambda(x)\right)$ is norm-continuous.

Proof. $\left\|P \circ \delta\left[\lambda(f)^{*} a^{*} a \lambda(f)(\lambda(x)-\lambda(y))\right]\right\| \leqslant\|a\|^{2}\|f\|_{2}\left\|f_{x}-f_{y}\right\|_{2}$ where $f_{x}(z)=\Delta\left(x^{-1}\right) f\left(z x^{-1}\right)$ for $x, z \in G$. If $x \rightarrow y,\left\|f_{x}-f_{y}\right\|_{2} \rightarrow 0$, which proves the continuity.

LeMma 3.3. For $f \in C_{00}(G), a \in \mathfrak{A}$ we have $P \circ \delta\left(\lambda(f)^{*} a^{*} a \lambda(f)\right) \in M(\mathfrak{R})$.

Proof. Suppose $b \in \mathfrak{A}$ and let $U$ be a neighbourhood of $e$ in $G$ such that for $x \in U$,

$$
\left\|P \circ \delta\left(\lambda(f)^{*} a^{*} a(\lambda(f)-\lambda(f) \lambda(x))\right)\right\|<\varepsilon \text { and }\left\|\lambda\left(x^{-1}\right) b-b\right\|<\varepsilon .
$$

Take $g \in C_{00}(G)^{+}$with support in $U$ and with $\int_{G} g(x) d x=1$. If we identify $g$ with its image in $C_{r}^{*}(G)$ we have from Lemma 1.5 that

$$
P\left[\delta\left(\lambda(f)^{*} a^{*} a \lambda(f)\right)(b \otimes g)\right] \in \mathfrak{A} .
$$

Furthermore

$$
\begin{aligned}
&\left\|P \circ \delta\left(\lambda(f)^{*} a^{*} a \lambda(f)\right) b-P\left[\delta\left(\lambda(f)^{*} a^{*} a \lambda(f)\right)(b \otimes g)\right]\right\| \\
& \leqslant\left\|\int_{G} g(x) P \circ \delta\left(\lambda(f)^{*} a^{*} a \lambda(f)\right)\left(b-\lambda\left(x^{-1}\right) b\right) d x\right\| \\
&+\left\|\int_{G} g(x) P \circ \delta\left[\lambda(f)^{*} a^{*} a(\lambda(f)-\lambda(f) \lambda(x))\right] \lambda\left(x^{-1}\right) b d x\right\| \\
& \leqslant \varepsilon\left(\left\|P \circ \delta\left(\lambda(f)^{*} a^{*} a \lambda(f)\right)\right\|+\|b\|\right) .
\end{aligned}
$$

Thus $P \circ \delta\left(\lambda(f)^{*} a^{*} a \lambda(f)\right) b \in \mathfrak{A}$, and in a similar way we can show that also $b P \circ \delta\left(\lambda(f)^{*} a^{*} a \lambda(f)\right) \in \mathfrak{A}$ for all $b \in \mathfrak{A}$, hence $P \circ \delta\left(\lambda(f)^{*} a^{*} a \lambda(f)\right) \in$ $M(\mathfrak{U})$. 
Lemma 3.4. $P \circ \delta\left(\lambda(f)^{*} a^{*} a \lambda(f)\right) \lambda(g) \in \mathfrak{A}$ for $a \in \mathfrak{A}, f, g \in C_{00}(G)$.

Proof. Let $\varepsilon>0$ and take a neighbourhood $U$ of $e$ in $G$ such that for $x \in U$

$$
\left\|P \circ \delta\left[\lambda(f)^{*} a^{*} a(\lambda(f)-\lambda(f) \lambda(x))\right]\right\|<\varepsilon \text { and }\left\|g-L_{x^{-1}} g\right\|_{1}<\varepsilon
$$

where as before \|\|$_{1}$ is the $L^{1}$-norm on $C_{00}(G)$ and $L_{x^{-1}} g(y)=g(x y)$ for $x, y \in G$. Now take $\alpha \in C_{00}(G)^{+}$with support in $U$ and with $\int \alpha(x) d x=1$. From Lemma 1.5

$$
P\left[\delta\left(\lambda(f)^{*} a^{*} a \lambda(f)\right)(\lambda(g) \otimes \alpha)\right] \in \mathfrak{A}
$$

and

$$
\begin{aligned}
& \left\|P \circ \delta\left(\lambda(f)^{*} a^{*} a \lambda(f)\right) \lambda(g)-P\left[\delta\left(\lambda(f)^{*} a^{*} a \lambda(f)\right)(\lambda(g) \otimes \alpha)\right]\right\| \\
& \leqslant\left\|\int \alpha(x) P \circ \delta\left(\lambda(f)^{*} a^{*} a \lambda(f)\right)\left(\lambda(g)-\lambda\left(x^{-1}\right) \lambda(g)\right) d x\right\| \\
& \quad+\left\|\int \alpha(x) P \circ \delta\left[\lambda(f)^{*} a^{*} a(\lambda(f)-\lambda(f) \lambda(x))\right] \lambda\left(x^{-1}\right) \lambda(g) d x\right\| \\
& \leqslant \varepsilon\left(\left\|P \circ \delta\left(\lambda(f)^{*} a^{*} a \lambda(f)\right)\right\|+\|\lambda(g)\|\right) .
\end{aligned}
$$

Thus $P \circ \delta\left(\lambda(f)^{*} a^{*} a \lambda(f)\right) \lambda(g) \in \mathfrak{A}$ and of course also

$$
\lambda(g) P \circ \delta\left(\lambda(f)^{*} a^{*} a \lambda(f)\right) \in \mathfrak{A} .
$$

Lemma 3.5. For $f \in C_{00}(G), a \in \mathfrak{A}$ let $b=P \circ \delta\left(\lambda(f)^{*} a^{*} a \lambda(f)\right)$, then the map $x \rightarrow \lambda(x) b \lambda\left(x^{-1}\right)$ is norm-continuous.

Proof. If $g \in C_{00}(G), x \in G$ define $g_{x}$ as in Lemma 3.2, then

$$
\begin{aligned}
\operatorname{tr}\left(x^{-1} g^{*} g x\right) & =\operatorname{tr}\left(g_{x}^{*} g_{x}\right)=\left\langle g_{x}, g_{x}\right\rangle=\Delta\left(x^{-1}\right)\langle g, g\rangle \\
& =\Delta\left(x^{-1}\right) \operatorname{tr}\left(g^{*} g\right) .
\end{aligned}
$$

From this it follows that for $c \in \mathfrak{U}^{* *} \otimes_{\mathrm{alg}} C_{00}(G)$

$$
P\left[\left(I \otimes x^{-1}\right) c(I \otimes x)\right]=\Delta\left(x^{-1}\right) P(c) .
$$

By a continuity argument similar to the one given in Lemma 2.9 we will have that

$$
P\left[\left(I \otimes x^{-1}\right) c(I \otimes x)\right]=\Delta\left(x^{-1}\right) P(c) \quad \text { for all } c \in\left(\mathfrak{U}^{* *} \otimes \mathcal{L}(G)\right)_{\mathrm{tr}} .
$$

So in particular

$$
\begin{aligned}
\lambda(x) b \lambda\left(x^{-1}\right) & =\lambda(x) P \circ \delta\left(\lambda\left(f^{*}\right) a^{*} a \lambda(f)\right) \lambda\left(x^{-1}\right) \\
& =P\left[\left(I \otimes x^{-1}\right) \delta\left(\lambda(x) \lambda(f)^{*} a^{*} a \lambda(f) \lambda\left(x^{-1}\right)\right)(I \otimes x)\right] \\
& =\Delta\left(x^{-1}\right) P \circ \delta\left[\lambda\left(f_{x^{-1}}\right)^{*} a^{*} a \lambda\left(f_{x^{-1}}\right)\right] .
\end{aligned}
$$


The right regular representation of $G$ on $L^{2}(G)$ is defined by $R_{x} f(y)=$ $\Delta(x)^{1 / 2} f(y x)$. So $R_{x} f=\Delta(x)^{-1 / 2} f_{x^{-1}}$, and therefore

$$
\lambda(x) b \lambda\left(x^{-1}\right)=P \circ \delta\left[\lambda\left(R_{x} f\right)^{*} a^{*} a \lambda\left(R_{x} f\right)\right]
$$

and

$$
\begin{aligned}
\left\|\lambda(x) b \lambda\left(x^{-1}\right)-b\right\| \leqslant & \left\|P \circ \lambda\left[\lambda\left(R_{x} f-f\right)^{*} a^{*} a \lambda\left(R_{x} f\right)\right]\right\| \\
& +\left\|P \circ \delta\left[\lambda(f)^{*} a^{*} a \lambda\left(R_{x} f-f\right)\right]\right\| \\
\leqslant & \left\|R_{x} f-f\right\|_{2}\left(\left\|R_{x} f\right\|_{2}+\|f\|_{2}\right) \cdot\|a\|^{2} \\
& =2\left\|R_{x} f-f\right\|_{2}\|f\|_{2} \cdot\|a\|^{2} .
\end{aligned}
$$

So if $x \rightarrow e$ this expression converges to zero.

Proof of Theorem 3. Take $A=\{a \in M(\mathfrak{A}) \mid$ (3.6), (3.7) and (3.8) hold $\}$, this is a $C^{*}$-subalgebra of $M(\mathfrak{A})$. Define $\rho: G \rightarrow \operatorname{Aut}(A)$ by $\rho_{x}(a)=$ $\lambda(x) a \lambda\left(x^{-1}\right)$, so $\rho$ is a norm-continuous automorphic representation of $G$ over $A$. From the Lemmas 2.8, 3.3, 3.4 and 3.5 it follows that

$$
P \circ \delta\left(\lambda(f)^{*} a^{*} a \lambda(f)\right) \in A \quad \text { for } a \in \mathfrak{A}, f \in C_{00}(G) .
$$

Now let $\mathfrak{B}=C_{r}^{*}(A, \rho, G)$, then $\mathfrak{B}$ acts on $L^{2}(G, H)$ if $\mathfrak{A}$ acts on a Hilbert space $H$. Define a unitary operator $U$ over $L^{2}(G, H)$ by

$$
U f(x)=\lambda(x) f(x) \quad \text { for } x \in G, f \in L^{2}(G, H) .
$$

$\mathfrak{B}$ is the $C^{*}$-algebra generated by $\left\{\tilde{\rho}(\varphi) \mid \varphi \in C_{00}(G, A)\right\}$ over $L^{2}(G, H)$ where

$$
\begin{aligned}
& \tilde{\rho}(\varphi) f(x)=\int_{G} \lambda\left(x^{-1}\right) \varphi(y) \lambda(x) f\left(y^{-1} x\right) d y \\
& \quad \text { for } \varphi \in C_{00}(G, A), f \in L^{2}(G, H) .
\end{aligned}
$$

Then we will have

$$
U \tilde{\rho}(\varphi) U^{*} f(x)=\int_{G} \varphi(y) \lambda(y) f\left(y^{-1} x\right) d y .
$$

If $a \in A, g \in C_{00}(G)$, let $a \otimes g$ denote the element in $C_{00}(G, A)$ defined by $a \otimes g(x)=g(x) a$. Then

$$
U \tilde{\rho}(a \otimes g) U^{*} f(x)=\int_{G} g(y) a \lambda(y) f\left(y^{-1} x\right) d y=(a \otimes I) \delta \circ \lambda(g) f(x)
$$

so $U \tilde{\rho}(a \otimes g) U^{*}=(a \otimes I) \delta \circ \lambda(g)=\delta(a \lambda(g)) \in \delta(\mathfrak{H})$ for $a \in A, \quad g \in$ $C_{00}(G)$. Since $\left\{\tilde{\rho}(a \otimes g) \mid a \in A, g \in C_{00}(G)\right\}$ generates $\mathfrak{B}$, we have $U \mathscr{B} U^{*} \subseteq$ $\delta(\mathfrak{A})$, and the map $b \rightarrow U b U^{*}$ is a ${ }^{*}$-isomorphism of $\mathfrak{B}$ into $\delta(\mathfrak{U})$. In order to prove that $U \mathscr{B} U^{*}=\delta(\mathfrak{A})$ we shall need 
LEMMA 3.6. Suppose $a \in \mathfrak{A}, f \in C_{00}(G)$ and $\alpha \in B_{r}(G) \cap C_{00}(G)$. Let $\tilde{\alpha} \in C_{00}(G, A)$ be defined by

$$
\tilde{\alpha}(x)=\Delta\left(x^{-1}\right) \alpha(x) P \circ \delta\left(\lambda(f)^{*} a^{*} a \lambda(f) \lambda\left(x^{-1}\right)\right) .
$$

Then we will have for all $\varphi \in \mathfrak{U} *$

$$
\left\langle\delta^{-1}\left(U \tilde{\rho}(\tilde{\alpha}) U^{*}\right), \varphi\right\rangle=\left\langle\lambda(f)^{*} a^{*} a \lambda(f), \varphi \alpha\right\rangle .
$$

Proof. First note that by Lemma 3.2, $\tilde{\alpha}$ is in fact in $C_{00}(G, A)$. We may assume that $\varphi(a)=\langle a \xi, \eta\rangle$ and $\alpha(x)=\left\langle L_{x} \beta, \gamma\right\rangle$ for suitable vectors $\xi, \eta \in$ $H$ and $\beta, \gamma \in C_{00}(G)$, and from (3.10) we see that

$$
\begin{aligned}
\delta^{-1}\left(U \tilde{\rho}(\psi) U^{*}\right) & =\delta^{-1}\left(\int_{G} \psi(y) \lambda(y) \otimes y d y\right) \\
& =\int_{G} \psi(y) \lambda(y) d y \quad \text { for } \psi \in C_{00}(G, A) .
\end{aligned}
$$

So for $\varphi \in \mathfrak{A}^{*}$

$$
\begin{aligned}
\left\langle\lambda(f)^{*} a^{*} a \lambda(f), \varphi \alpha\right\rangle=\left\langle\delta\left(\lambda(f)^{*} a^{*} a \lambda(f)\right), \varphi \otimes \alpha\right\rangle & \\
& =\left\langle\delta\left(\lambda(f)^{*} a^{*} a \lambda(f)\right)(\xi \otimes \beta), \eta \otimes \gamma\right\rangle \quad(2.25) \\
& =\left\langle\delta\left(\lambda(f)^{*} a^{*} a\right) R_{\beta} U(\xi \otimes f), \eta \otimes \gamma\right\rangle \\
& =\left\langle R_{\beta} \delta\left(\lambda(f)^{*} a^{*} a\right) U(\xi \otimes f), \eta \otimes \gamma\right\rangle \\
& =\int_{G} \int_{G} \beta\left(x^{-1} y\right)\left\langle\delta\left(\lambda(f)^{*} a^{*} a\right) U(\xi \otimes f)(x),(\eta \otimes \gamma)(y)\right\rangle d x d y(2.21) \\
& =\int_{G} \int_{G} \Delta\left(x^{-1}\right) \beta\left(x^{-1} y\right)\left\langle P \circ \delta\left(\lambda(f)^{*} a^{*} a \lambda(f) \lambda\left(x^{-1}\right)\right)\right. \\
& =\int_{G} \int_{G} \Delta\left(x^{-1}\right) \beta\left(x^{-1} y\right) \overline{\gamma(y)}\left\langle P \circ \delta\left(\lambda(f)^{*} a^{*} a \lambda(f) \lambda\left(x^{-1}\right)\right)\right. \\
& =\int_{G} \Delta\left(x^{-1}\right) \alpha(x)\left\langle P \circ \delta\left(\lambda(f)^{*} a^{*} a \lambda(f) \lambda\left(x^{-1}\right)\right) \lambda(x) \xi, \eta\right\rangle d x \\
= & \int_{G}\langle\tilde{\alpha}(x) \lambda(x) \xi, \eta\rangle d x=\left\langle\delta^{-1}\left(U \tilde{\rho}(\tilde{\alpha}) U^{*}\right) \xi, \eta\right\rangle \\
= & \left\langle\delta^{-1}\left(U \tilde{\rho}(\tilde{\alpha}) U^{*}\right), \varphi\right\rangle .
\end{aligned}
$$

This proves (3.11).

To prove Theorem 3, we must show that $U \mathscr{B} U^{*}=\delta(\mathfrak{A})$, i.e. that $\delta^{-1}\left(U \mathfrak{B} U^{*}\right)=\mathfrak{A}$. Suppose this is not true, then there is a $\varphi \in \mathfrak{A}^{*}$ with $\varphi \neq 0$, 
$\varphi\left(\delta^{-1}\left(U \mathscr{B} U^{*}\right)\right)=\{0\}$. Since $\delta$ is nondegenerate there is an $\alpha \in B_{r}(G)$ with $\varphi \alpha \neq 0$, so there is an $a \in \mathfrak{A}$ with $\varphi \alpha(a)=\langle\delta(a), \varphi \otimes \alpha\rangle \neq 0$. If $\left\{g_{i}\right\}$ is a net of functions from $C_{00}(G)$ such that the corresponding elements of $C_{r}^{*}(G)$ give a bounded approximate identity, it is not too difficult to prove that $b_{i}=\left(I \otimes g_{i}\right) \delta(a)\left(I \otimes g_{i}\right)$ converges to $\delta(a)$ in the multiplier topology on $M\left(\mathfrak{A} \otimes C_{r}^{*}(G)\right)$. So $\left\langle b_{i}, \varphi \otimes \alpha\right\rangle \neq 0$ for some $i$, and since $b_{i} \in \mathfrak{A} \otimes C_{r}^{*}(G)$ this expression can be approximated with $\left\langle b_{i}, \varphi \otimes \beta\right\rangle$ where $\beta \in B_{r}(G) \cap$ $C_{00}(G)$, so there is a $\beta \in B_{r}(G) \cap C_{00}(G)$ with

$$
0 \neq\left\langle b_{i}, \varphi \otimes \beta\right\rangle=\left\langle\left(I \otimes g_{i}\right) \delta(a)\left(I \otimes g_{i}\right), \varphi \otimes \beta\right\rangle=\langle\delta(a), \varphi \otimes \gamma\rangle .
$$

Here $\gamma \in B_{r}(G)$ is defined by $\gamma(x)=\beta\left(g_{i} x g_{i}\right)$ for $x \in C_{r}^{*}(G)$, so $\gamma$ also has compact support. So there is an $\alpha \in B_{r}(G) \cap C_{00}(G)$ such that $\varphi \alpha(a) \neq 0$.

linspan $\left\{\lambda(f)^{*} a^{*} a \lambda(f) \mid a \in \mathfrak{A}, f \in C_{00}(G)\right\}$ is norm-dense in $\mathfrak{A}$, so there is $a \in \mathfrak{A}$ and $f \in C_{00}(G)$ with $\left\langle\lambda(f)^{*} a^{*} a \lambda(f), \varphi \alpha\right\rangle \neq 0$. But according to (3.11)

$$
\left\langle\lambda(f)^{*} a^{*} a \lambda(f), \varphi \alpha\right\rangle=\left\langle\delta^{-1}\left(U \tilde{\rho}(\tilde{\alpha}) U^{*}\right), \varphi\right\rangle=0,
$$

a contradiction. So $\delta^{-1}\left(U \mathscr{B} U^{*}\right)=\mathfrak{A}$. In order to complete the proof of Theorem 3 we have to show that $\delta$ is automatically nondegenerate if $G$ is discrete or amenable, and this is done in the next two lemmas.

LemMa 3.7. If $G$ is a discrete group, the nondegeneracy of $\delta$ in Theorem 3 follows from the other assumptions.

Proof. If $G$ is discrete, $C_{r}^{*}(G)$ has an identity so

$$
\tilde{M}\left(\mathfrak{A} \otimes C_{r}^{*}(G)\right)=\mathfrak{A} \otimes C_{r}^{*}(G) .
$$

Suppose we have $\varphi \in \mathfrak{A}^{*}, a \in \mathfrak{A}$ with $\varphi(a) \neq 0 . \delta(a) \in \mathfrak{A} \otimes C_{r}^{*}(G)$ so there are sequences $\left\{a_{i}\right\}$ from $\mathfrak{U}$ and $\left\{x_{i}\right\}$ from $G$ with $x_{i} \neq x_{j}$ for $i \neq j$ such that

$$
\begin{aligned}
\delta(a)=\sum_{i=1}^{\infty} a_{i} & \otimes x_{i} \quad \text { (norm-convergence) } . \\
\sum_{i=1}^{\infty} \delta\left(a_{i}\right) \otimes x_{i} & =(\delta \otimes i) \delta(a)=\left(i \otimes \delta_{G}\right) \delta(a) \\
& =\sum_{i=1}^{\infty} a_{i} \otimes x_{i} \otimes x_{i},
\end{aligned}
$$

so $\delta\left(a_{i}\right)=a_{i} \otimes x_{i}$ for all $i$, thus $\delta(a)=\sum_{i=1}^{\infty} \delta\left(a_{i}\right)$ and $a=\sum_{i=1}^{\infty} a_{i}$. Since $\varphi(a) \neq 0, \varphi\left(a_{i}\right) \neq 0$ for some $i$. Take $\alpha \in B_{r}(G)$ such that $\alpha\left(x_{i}\right)=1, \alpha\left(x_{j}\right)=$ 
0 for $j \neq i$ (such an $\alpha$ will exist) then

$$
\begin{aligned}
\langle a, \varphi \alpha\rangle & =\left\langle\sum_{j=1}^{\infty} a_{j} \otimes x_{j}, \varphi \otimes \alpha\right\rangle \\
& =\sum_{j=1}^{\infty} \varphi\left(a_{j}\right) \alpha\left(x_{j}\right)=\varphi\left(a_{i}\right) \neq 0 .
\end{aligned}
$$

So $\varphi \alpha \neq 0$ and the lemma is proved.

Note that we now almost have given the simple proof possible for Theorem 3 when $G$ is discrete. We just proved that each $a \in \mathfrak{A}$ can be written $a=\sum_{i=1}^{\infty} a_{i}$ with $\delta\left(a_{i}\right)=a_{i} \otimes x_{i}, x_{i} \in G$. Therefore $a_{i} \lambda\left(x_{i}^{-1}\right) \in A$, so each $a_{i}$ and therefore also $a$ is in $\delta^{-1}\left(U \mathscr{B} U^{*}\right)$, using the terminology in the proof of Theorem 3. So $C_{r}^{*}(A, \rho, G) \cong \mathfrak{A}$.

LEMMA 3.8. If $G$ is an amenable locally compact group, the nondegeneracy of $\delta$ in Theorem 3 follows from the other assumptions.

Proof. If $G$ is amenable $C^{*}(G)=C_{r}^{*}(G)$ and $B_{r}(G)=B(G)$ so 1-the function equal to 1 everywhere on $G$-is in $B_{r}(G)$. It obviously suffices to prove that $\varphi 1=\varphi$ for all $\varphi \in \mathfrak{A}^{*}$.

If $c \in M\left(\mathfrak{A} \otimes C_{r}^{*}(G)\right)$ and $\alpha \in B_{r}(G)$ we can define $P_{\alpha}(c) \in \mathfrak{A}^{* *}$ by the formula

$$
\left\langle\varphi, P_{\alpha}(c)\right\rangle=\langle c, \varphi \otimes \alpha\rangle \text { for } \varphi \in \mathfrak{U}^{*} \text {. }
$$

As before $\varphi \otimes \alpha$ is extended to a linear functional on $M\left(\mathfrak{A} \otimes C_{r}^{*}(G)\right)$ continuous in the multiplier topology. If $c \in \mathfrak{A} \otimes C_{r}^{*}(G), P_{\alpha}(c) \in \mathfrak{A}$, this is in fact the situation considered by J. Tomiyama in [29]. So if we take $a \in \mathfrak{A}$, $b \in C_{r}^{*}(G)$ we have that $P_{\alpha}((I \otimes b) \delta(a)) \in \mathfrak{A}$. For $\varphi \in \mathfrak{U}^{*}$ we have

$$
\left\langle\varphi, P_{\alpha}((I \otimes b) \delta(a))\right\rangle=\langle(I \otimes b) \delta(a), \varphi \otimes \alpha\rangle=\langle\delta(a), \varphi \otimes b \alpha\rangle
$$

where $b \alpha \in B_{r}(G)$ is defined by

$$
\langle x, b \alpha\rangle=\langle b x, \alpha\rangle \text { for all } x \in C_{r}^{*}(G) .
$$

If we take $\alpha=1$ we will get (since 1 is a character) that

$$
\langle x, b 1\rangle=\langle b x, 1\rangle=\langle x, 1\rangle\langle b, 1\rangle \text {, i.e. } b 1=\langle b, 1\rangle 1 \text {. }
$$

So by taking a $b \in C_{r}^{*}(G)$ with $\langle b, 1\rangle=1$ (note that such an element exists) we have $b 1=1$ and $\left\langle\varphi, P_{1}((I \otimes b) \delta(a))\right\rangle=\langle\delta(a), \varphi \otimes 1\rangle=\left\langle\varphi, P_{1}(\delta(a))\right\rangle$. So $P_{1}(\delta(a))=P_{1}((I \otimes b) \delta(a)) \in \mathfrak{A}$. Thus

$$
\begin{aligned}
\left\langle\delta\left(P_{1}(\delta(a))\right),\right. & \otimes \otimes\rangle=\left\langle P_{1}(\delta(a)), \varphi \alpha\right\rangle \\
& =\langle\delta(a), \varphi \alpha \otimes 1\rangle=\langle a, \varphi \alpha 1\rangle=\langle a, \varphi \alpha\rangle \\
& =\langle\delta(a), \varphi \otimes \alpha\rangle \text { for all } \varphi \in \mathfrak{U}^{*}, \alpha \in B_{r}(G) .
\end{aligned}
$$

Thus $\delta\left(P_{1}(\delta(a))\right)=\delta(a)$ and since both $a$ and $P_{1}(\delta(a))$ are in $\mathfrak{A}$ and $\delta$ is 
one-to-one on $\mathfrak{A}$ we have $P_{1}(\delta(a))=a$. If we now have $\varphi \in \mathfrak{U}^{*}$ and $a \in \mathfrak{U}$, then

$$
\langle a, \varphi\rangle=\left\langle P_{1}(\delta(a)), \varphi\right\rangle=\langle\delta(a), \varphi \otimes 1\rangle=\langle a, \varphi 1\rangle .
$$

So $\varphi 1=\varphi$ for all $\varphi \in \mathfrak{A}^{*}$ and the lemma is proved.

REMARK. Since the nondegeneracy of $\delta$ is automatically fulfilled both if the group $G$ is amenable and if $G$ is discrete, one would conjecture that this is true in general. The author has been unable to prove this, the proofs of Lemma 3.7 and Lemma 3.8 are quite different, in the amenable case the fact that $B_{r}(G)$ has a unit is used, if $G$ is discrete one uses that $\delta(\mathfrak{A}) \subset \mathfrak{A} \otimes$ $C_{r}^{*}(G)$.

$C^{*}$-crossed products over an abelian group. If the group $G$ is abelian, we will have the following $C^{*}$-algebraic version of Theorem 2:

THEOREM 4. Suppose $\mathfrak{A}$ is a $C^{*}$-algebra and $G$ a locally compact abelian group. Then $\mathfrak{A}$ is isomorphic to a $C^{*}$-crossed product $C^{*}(A, \rho, G)$ for some norm-continuous automorphic representation $\rho$ of $G$ over a $C^{*}$-algebra $A$ if and only if there is a continuous homomorphism $\lambda: G \rightarrow M(\mathfrak{A})_{u}$ and a normcontinuous automorphic representation $\tau$ of $\hat{G}$ over $\mathfrak{A}$ such that

$$
\tau_{\gamma}(\lambda(x))=\gamma(x) \lambda(x) \text { for } x \in G, \gamma \in \hat{G} .
$$

$A$ and $\rho$ are uniquely determined by $\lambda$ and $\tau: \rho_{x}(a)=\lambda(x) a \lambda\left(x^{-1}\right)$,

$$
\begin{array}{r}
A=\left\{a \in M(\mathfrak{A}) \mid \tau_{\gamma}(a)=\text { a for all } \gamma \in \hat{G}, a \lambda(f) \text { and } \lambda(f) a \in \mathfrak{A}\right. \\
\text { for all } \left.f \in C_{00}(G), x \rightarrow \rho_{x}(a) \text { is norm-continuous }\right\} .
\end{array}
$$

Proof. Since an abelian group in particular is amenable we will have $C_{r}^{*}(A, \rho, G) \cong C^{*}(A, \rho, G)$. If $(A, \rho, G)$ is given and $\mathfrak{A}=C^{*}(A, \rho, G), \tau: \hat{G}$ $\rightarrow$ Aut $(\mathfrak{A})$ is defined exactly as in Theorem $2 . \mathfrak{A}$ acts on $L^{2}(G, H)$, and we define a unitary representation $U$ of $G$ on $L^{2}(G, H)$ by

$$
U_{\gamma} f(x)=\gamma(x) f(x) \text { for } f \in L^{2}(G, H), \gamma \in \hat{G}, x \in G .
$$

For $\varphi \in C_{00}(G, A)$ we will have

$$
\begin{aligned}
U_{\gamma} \tilde{\rho}(\varphi) U_{-\gamma} f(x) & =\gamma(x) \int \rho_{x^{-1}}(\varphi(y)) U_{-\gamma} f(x-y) d y \\
& =\int \gamma(y) \rho_{x^{-1}}(\varphi(y)) f(x-y) d y=\tilde{\rho}(\gamma \varphi) f(x)
\end{aligned}
$$

where $\gamma \varphi(y)=\gamma(y) \varphi(y)$ for $\gamma \in \hat{G}, y \in G$.

So $U_{\gamma} \mathfrak{A} U_{-\gamma}=\mathfrak{A}$ for all $\gamma \in \hat{G}$, and we can define $\tau: \hat{G} \rightarrow \operatorname{Aut}(\mathfrak{U})$ by

$$
\tau_{\gamma}(a)=U_{\gamma} a U_{-\gamma} \text { for } a \in \mathfrak{A}, \gamma \in \hat{G} \text {. }
$$

It is easy to see that the map $\gamma \rightarrow \tau_{\gamma}(\tilde{\rho}(\varphi))$ is continuous for $\varphi \in C_{00}(G, A)$, so $\tau$ will be a norm-continuous automorphic representation of $\hat{G}$ over $\mathfrak{A}$. If we 
define $\lambda: G \rightarrow M(\mathfrak{A})$ as before-i.e. $\lambda(x) f(y)=f(y-x)$ for $f \in L^{2}(G, H)$, $x, y \in G-i$ is easy to see that $\tau_{\gamma}(\lambda(x))=\gamma(x) \lambda(x)$ for $x \in G, \gamma \in \hat{G}$. The uniqueness part of Theorem 4, i.e. that $\tilde{\rho}(A)=\left\{a \in M(\mathfrak{A}) \mid \tau_{\gamma}(a)=a\right.$ for all $\gamma \in \hat{G}, a \lambda(f)$ and $\lambda(f) a \in \mathfrak{A}$ and $x \rightarrow \rho_{x}(a)$ is norm-continuous $\}$ now follows from the uniqueness part of Theorem 3 together with (2.30).

Conversely, suppose we have given a triple $(\mathfrak{A}, \lambda, \tau)$ satisfying the assumptions in Theorem 4. We are going to use Theorem 3 so we shall construct a ${ }^{*}$-isomorphism $\delta: \mathfrak{A} \rightarrow \tilde{M}\left(\mathfrak{A} \otimes C_{r}^{*}(G)\right)$ satisfying the requirements in Theorem 3. For $G$ abelian $C_{r}^{*}(G)=C^{*}(G)=C_{0}(\hat{G})$, and we have seen that $\tilde{M}\left(\mathfrak{A} \otimes C_{0}(\hat{G})\right) \cong C B(\hat{G}, \mathfrak{A}) . \quad \delta: \mathfrak{A} \rightarrow C B(\hat{G}, \mathfrak{A})$ is then simply defined by $\delta(a)(\gamma)=\tau_{\gamma}(a)$. Obviously $\delta(a) \in C B(\hat{G}, \mathfrak{A})$, and the map $a \rightarrow$ $\delta(a)$ is a ${ }^{*}$-isomorphism.

$$
\delta(\lambda(x))(\gamma)=\tau_{\gamma}(\lambda(x))=\gamma(x) \lambda(x)=(\lambda(x) \otimes \hat{x})(\gamma)
$$

for $x \in G, \gamma \in \hat{G}$ where $\hat{x}$ is the element of $M\left(C^{*}(G)\right)=C B(\hat{G})$ defined by $\hat{x}(\gamma)=\gamma(x)$. So (3.4) holds.

$\tilde{M}\left(C^{*}(G) \otimes C^{*}(G)\right) \cong C B\left(\hat{G}, C_{0}(\hat{G})\right)$ and the corresponding map $\delta_{G}$ : $C_{0}(\hat{G}) \rightarrow C B\left(\hat{G}, C_{0}(\hat{G})\right) \subseteq C B(\hat{G} \times \hat{G})$ is given by $\delta_{G}(\varphi)(\alpha, \beta)=\varphi(\alpha+\beta)$ for $\alpha, \beta \in \hat{G}, \varphi \in C_{0}(\hat{G})$. This was proved in Theorem 2 and we also showed that $(\delta \otimes i) \delta(a)=\left(i \otimes \delta_{G}\right) \delta(a)$ for $a \in \mathfrak{A}$, so (3.5) also holds. If $a \in M(\mathfrak{A})$, we have that $\delta(a)=a \otimes I \Leftrightarrow \tau_{\gamma}(a)=a$ for all $\gamma \in \hat{G}$, so Theorem 4 now follows from Theorem 3 .

A covariant system $(A, \rho, G)$ over an abelian group $G$ thus naturally gives us a new covariant system $(\mathfrak{A}, \tau, \hat{G})$ over the dual group $\hat{G}$ where $\mathfrak{A}=$ $C^{*}(A, \rho, G)$. H. Takai has proved in [33, Theorem 3.4] that $C^{*}(\mathfrak{A}, \tau, \hat{G}) \cong A$ $\otimes C C\left(L^{2}(G)\right)$ and that the natural automorphic representation $\hat{\rho}$ of $G$ on $C^{*}(\mathfrak{A}, \tau, \hat{G})$ corresponds to $\rho_{x} \otimes \operatorname{ad}(-x)$. Here $C C\left(L^{2}(G)\right)$ is the $C^{*}$-algebra of compact operators on $L^{2}(G)$ and $\operatorname{ad}(x)$ is the automorphism defined by $\operatorname{ad}(x) a=x a x^{-1}$ for $x \in G, a \in C C\left(L^{2}(G)\right)$.

\section{BIBLIOGRAPHY}

1. C. A. Akemann, G. K. Pedersen and J. Tomiyama, Multipliers on $C^{*}$-algebras, J. Functional Analysis 13 (1973), 277-301.

2. H. Araki and J. Woods, A classification of factors, Publ. Res. Inst. Math. Sci. 4 (1968), 51-130.

3. R. C. Busby, Double centralizers and extensions of $C^{*}$-algebras, Trans. Amer. Math. Soc. 132 (1968), 79-99. MR 37 \#770.

4. F. Combes, Poids sur une $C^{*}$-algèbre, J. Math. Pures Appl. (9) 47 (1968), 57-100. MR 38 \#5016.

5. __ Poids associè à une algèbre hilbertienne à gauche, Compositio Math. 23 (1971), 49-77. MR 44 \# 5786.

6. A. Connes, Une classification de facteurs de type III, Ann. Sci. École Norm. Sup. 6 (1973), 133-252. MR 49 \#5865.

7. A. Derighetti, Some results on the Fourier-Stieltjes algebra of a locally compact group, Comment. Math. Helv. 45 (1970), 219-228. 
8. J. Dixmier, Les algèbres d'opérateurs dans l'espace hilbertien, 2nd ed., Gauthier-Villars, Paris, 1969.

9 \# 1404. Les $C^{*}$-algèbres et leurs représentations, Gauthier-Villars, Paris, 1969. MR 30

10. S. Doplicher, D. Kastler and D. Robinson, Covariance algebras in field theory and statistical mechanics, Comm. Math. Phys. 3 (1966), 1-28.

11. E. G. Effros and F. Hahn, Locally compact transformation groups and $C^{*}$-algebras, Mem. Amer. Math. Soc. No. 75 (1967). MR 37 \#2895.

12. E. G. Effros and E. C. Lance, Tensor products of operator algebras, Advances in Math. 25 (1977), $1-34$.

12. E. G. Effros and E. C. Lance, Tensor products of operator algebras (preprint).

13. J. Ernest, The enveloping algebra of a covariant system, Comm. Math. Phys. 17 (1970), 61-74. MR 43 \# 1553.

14. $A$ A duality theorem for the automorphism group of a covariant system, Comm. Math. Phys. 17 (1970), 75-90. MR 42 \#8298.

15. J. M. G. Fell, An extension of Mackey's method to Banach *-algebraic bundles, Mem. Amer. Math. Soc. No. 90 (1969).

16. G. K. Pedersen and M. Takesaki, The Radon-Nikodym theorem for von Neumann algebras, Acta Math. 130 (1973), 53-87.

17. R. T. Powers, Representations of uniformly hyperfinite algebras and their associated von Neumann rings, Ann. of Math. (2) 86 (1967), 138-171. MR 36 \# 1989.

18. M. Rieffel, Induced representations of $C^{*}$-algebras, Advances in Math. 13 (1974), 176-257. MR 50 \#5489.

19. W. Rudin, Fourier analysis on groups, Interscience, New York, 1967.

20. S. Sakai, $C^{*}$-algebras and $W^{*}$-algebras, Springer-Verlag, Berlin and New York, 1971.

21. M. E. Sweedler, Hopf algebras, Benjamin, New York, 1969. MR 40 \#705.

22. M. Takesaki, Covariant representations of $C^{*}$-algebras and their locally compact groups, Acta Math. 119 (1967), 273-303.

23. __ A characterization of group algebras as a converse of Tannaka-Stinespring-Tatsuuma duality, Amer. J. Math. 91 (1969), 529-564.

24. __ Tomita's theory of modular Hilbert algebras and its applications, Lecture Notes in Math., vol. 128, Springer-Verlag, Berlin and New York, 1970.

25. __ A liminal crossed product of a uniformly hyperfinite $C^{*}$-algebra by a compact abelian group, J. Functional Analysis 7 (1971), 140-146.

26. , Duality and von Neumann algebras, Bull. Amer. Math. Soc. 77 (1971), 553-557. MR 46 \# 4225.

27. _ The structure of a von Neumann algebra with a homogeneous periodic state, Acta Math. 131 (1973), 79-122.

28. __ Duality for crossed products and structure of type III von Neumann algebras, Acta Math. 131 (1967), 249-310.

29. J. Tomiyama, Fubini type theorems to the tensor product of $C^{*}$-algebras, Tôhoku Math. J. 19 (1967), 213-226.

30. G. Zeller-Meier, Produits croisés d'une $C^{*}$-algèbre par une group d'automorphismes, J Math. Pures Appl. (9) 47 (1968), 101-239. MR 39 \#3329.

31. U. Haagerup, Operator valued weights and crossed products, Symposia Math. 20 (1976), 241-251.

32. H. Takai, Dualité dans les produits croisés de C*-algèbres, C. R. Acad. Sci. Paris 278 (1974), 1041-1043. MR 49 \#5863.

33. 25-39. On a duality for crossed products of $C^{*}$-algebras, J. Functional Analysis 19 (1975),

Department of Mathematics, University of Trondheim, 7000 Trondheim, Norway 